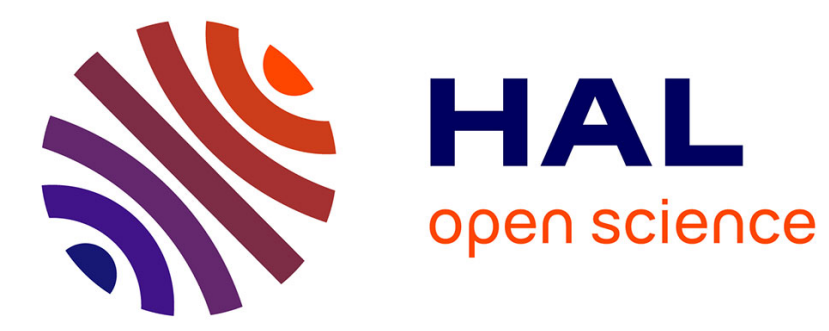

\title{
Utiliser une simulation vidéo 3D pour approcher les concepts de la cinématique relativiste : étude de cas
}

Clément Maisch, Isabelle Kermen, C. de Hosson, Etienne Parizot

\section{To cite this version:}

Clément Maisch, Isabelle Kermen, C. de Hosson, Etienne Parizot. Utiliser une simulation vidéo 3D pour approcher les concepts de la cinématique relativiste : étude de cas. RDST - Recherches en didactique des sciences et des technologies , 2015, 12, pp.141 - 174. 10.4000/rdst.1152 . hal-01663447

\section{HAL Id: hal-01663447 https://hal.science/hal-01663447}

Submitted on 18 Dec 2017

HAL is a multi-disciplinary open access archive for the deposit and dissemination of scientific research documents, whether they are published or not. The documents may come from teaching and research institutions in France or abroad, or from public or private research centers.
L'archive ouverte pluridisciplinaire HAL, est destinée au dépôt et à la diffusion de documents scientifiques de niveau recherche, publiés ou non, émanant des établissements d'enseignement et de recherche français ou étrangers, des laboratoires publics ou privés. 


\section{RDST}

Recherches en didactique des sciences et des

technologies

$12 \mid 2015$

L'expérimental en sciences, réel ou virtuel

\section{Utiliser une simulation vidéo 3D pour approcher les concepts de la cinématique relativiste : étude de cas}

Using a video simulation to approach concepts from relativistic kinematics: a case study

\section{Clément Maisch, Isabelle Kermen, Cécile de Hosson et Étienne Parizot}

\section{revues.org}

Édition électronique

URL : http://rdst.revues.org/1152

DOI : $10.4000 /$ rdst. 1152

ISSN : 2271-5649

\section{Éditeur}

ENS Éditions

\section{Édition imprimée}

Date de publication : 15 novembre 2015

Pagination : 141-174

ISBN : $978-2-84788-647-4$

ISSN : $2110-6460$

Référence électronique

Clément Maisch, Isabelle Kermen, Cécile de Hosson et Étienne Parizot, « Utiliser une simulation vidéo 3D pour approcher les concepts de la cinématique relativiste : étude de cas », RDST [En ligne],

12 | 2015, mis en ligne le 15 novembre 2017, consulté le 15 novembre 2017. URL : http:// rdst.revues.org/1152; DOI : 10.4000/rdst.1152 


\title{
Utiliser une simulation vidéo 3D pour approcher les concepts de la cinématique relativiste : étude de cas
}

\author{
Clément Maisch \\ Université Pierre-Mendès-France, Grenoble, IAE \\ Isabelle Kermen \\ Université d'Artois, LDAR \\ Cécile de Hosson \\ Université Paris-Diderot, LDAR \\ Étienne Parizot \\ Université Paris-Diderot, APC
}

RÉSUMÉ • L'observation des effets directement liés à la théorie de la relativité restreinte (TRR) sur des objets de la vie de tous les jours est impossible. En effet, de tels effets ne peuvent être attestés qu'en se déplaçant à des vitesses proches de celle de la lumière $c$. La réalité virtuelle (RV) permet de visualiser ces effets en utilisant des environnements à trois dimensions dans lesquels les valeurs de la vitesse de déplacement d'objets virtuels (ici, des palets de billard français) et d'observateurs (réels) immergés peuvent s'approcher à loisir de la vitesse de la lumière, ramenée au sein de la simulation à une valeur familière de $1 \mathrm{~m} / \mathrm{s}$. Dans cet article nous présentons les résultats d'une étude exploratoire se donnant pour objet l'évaluation de vidéos reproduisant certaines scènes produites pour l'environnement immersif. Cette étude a été conduite auprès de neuf étudiants de master enseignement physique-chimie amenés à enseigner la relativité restreinte. Les vidéos donnent à voir les observables que sont la non-simultanéité d'événements, la déformation des objets mis en scène, la modification de leur vitesse apparente. Les premiers résultats de cette étude permettent de saisir la manière dont ces observables mis en scène contribuent à la mobilisation d'explications de nature relativiste engageant, d'une part, la localisation spatio-temporelle des émissions lumineuses à partir des objets virtuels et celle de leur réception dans l'œil de l'observateur, d'autre part, la transformée de Lorentz.

MOTS-CLÉS • réalité augmentée, simulation, lumière, relativité restreinte, raisonnement de sens commun 


\begin{abstract}
The observation of effects directly connected to special relativity on objects of the daily life is impossible. Indeed, such effects can be attested only by moving at speeds close to that of the light c. Virtual reality (RV) allows to visualize these effects through 3D immersive environments in which the values of the speed of virtual moving objects (here, pucks) and of immersed observers are close to the speed of light, returned within the simulation to a familiar value of $1 \mathrm{~m} / \mathrm{s}$. In this article results of an exploratory study are presented. This study aims at evaluating the use of videos reproducing certain stages produced for the immersive environment. The first results of this study, conducted with nine students prospective physics and chemistry teachers (brought to teach special relativity) allow to seize the way that the staged observables (non-simultaneity of events, distortion of the pucks, modification of their apparent speed) contribute to the mobilization of explanations of relativist nature involving on the one hand, the spatio-temporal location of light emissions from the virtual objects and that of their receptions into the eye of the observer, on the other hand, Lorentz transformation.
\end{abstract}

KEYWORDS • augmented reality, simulation, light, special relativity, common-sense reasoning

\title{
1. Contexte de la recherche
}

\subsection{Le projet EVEILS}

La recherche que nous présentons s'inscrit dans le contexte du projet collaboratif et interdisciplinaire EVEILS (Environnements Virtuels pour l'Éducation et l'ILlustration Scientifiques) conduit entre 2009 et 2012 dans le but d'étudier l'impact de l'usage d'une plateforme de réalité virtuelle 3D - une CAVE', sur la compréhension des effets associés au cadre de la relativité restreinte ${ }^{2}$. Il s'agissait d'immerger des observateurs dans un environnement métaphorique (une salle de billard français) au sein duquel des objets (des palets de billards) et l'observateur peuvent se déplacer à une vitesse proche de la vitesse de la lumière, ajustable à volonté dans l'espace-temps simulé, et ramenée pour les besoins de l'expérience à $1 \mathrm{~m} / \mathrm{s}$ (voir figure 1 ).

1 Une CAVE (Cave Automatic Virtual Environment) est un système de projection de réalité virtuelle (ou RV) constitué d'un ensemble d'écrans (et éventuellement de générateurs de son) disposés autour de l'utilisateur, et formant un espace clos ou partiellement clos au sein duquel il peut se mouvoir à loisir. L'illusion de l'immersion est créée par la projection d'images de synthèse sur les écrans occupant le champ de vue de l'utilisateur, calculées en fonction de sa position et de la direction de son regard, conformément au point de vue qui en résulterait si cette position et cette orientation étaient réellement les siennes non pas dans la CAVE physique où se déroule l'expérience, mais dans l'environnement virtuel qu'elle simule. Cette immersion est donc couplée à un système de repérage (tracking) de la position et de l'orientation de la tête de l'utilisateur, et un système de lunettes 3D actives permet de reproduire la vision stéréoscopique habituelle en présentant à chaque œil les images calculées pour sa position propre.

2 EVEILS est un projet soutenu par l'Agence nationale de la recherche (ANR - programme blanc). 


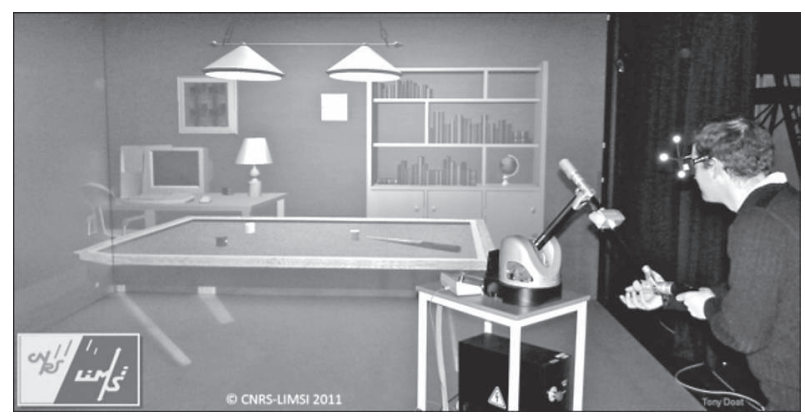

Fig. 1 : photographie de l'environnement métaphorique implémenté dans la CAVE du projet EVEILS L'observateur (à droite de la photographie) déclenche le mouvement du palet orange à l'aide d'un dispositif haptique qui commande la queue de billard; les mouvements des palets sont ensuite calculés en fonction des positions successives de l'observateur (repérées grâce aux capteurs localisés sur ses lunettes 3D) au sein de la scène virtuelle.

Les raisons du choix et la construction de la métaphore ayant d'ores et déjà fait l'objet de plusieurs publications (Doat, Parizot \& Vezien, 2011; de Hosson et al., 2012), nous allons nous concentrer ici sur l'usage qui en a été fait dans le contexte d'une enquête impliquant neuf étudiants de master MEEF première année se destinant au métier de professeur de physique-chimie (susceptibles d'enseigner des éléments de relativité restreinte : voir MEN, 2011). L'idée, à terme, est de promouvoir ce type d'outil en tant que support de formation disciplinaire mais également en tant que support d'alerte. En effet, Internet regorge de ressources pédagogiques donnant "à voir " les effets relativistes, ressources vers lesquelles les futurs enseignants risquent de se tourner si l'on considère la place accordée par l'institution scolaire aux activités documentaires dans les nouveaux programmes de lycée. Or, dans de nombreux cas, les ressources disponibles (vidéo, notamment) valorisent visuellement la contraction des longueurs alors que ce phénomène n'est pas directement observable en milieu relativiste. L'usage, en formation, d'une simulation de scènes relativistes doit permettre de sensibiliser les futurs enseignants au fait que la visualisation des effets relativistes ne se limite pas à la contraction des longueurs mais nécessite également la prise en compte de la durée mise par la lumière pour parcourir la distance d'un point-objet à l'œil, durée qui, dans la vie quotidienne, est considérée comme nulle. À ce stade de notre travail, la simulation proposée n'a pas vocation à être utilisée en classe.

\subsection{La relativité restreinte: de la théorie à la visualisation}

Lorsque l'on "met en image" un objet macroscopique et un observateur, en mouvement l'un par rapport à l'autre à des vitesses proches de celles de la lumière, la forme et la vitesse de l'objet apparaissent différentes de ce qui est habituellement perçu lorsque les vitesses de déplacement de ces objets ont des valeurs très faibles devant celles de la lumière (c'est-à-dire les vitesses de la vie quotidienne). Ces différences sont dues à deux effets conjugués : la prise en compte du caractère fini de la vitesse de la lumière et les effets liés à la géométrie particulière de l'espace-temps quadridimensionnel en relativité restreinte, et qu'on pourra qualifier de "minkovskiens". 


\subsubsection{Effet "minkovskien" : la contraction des longueurs}

Considérons deux référentiels inertiels $R$ et $R$ ', en mouvement rectiligne et uniforme l'un par rapport à l'autre selon une direction spatiale qu'on choisira comme le premier axe d'un système de coordonnées cartésiennes attaché à chacun de ces référentiels ("axe des $x$ "). On considère également deux observateurs, 0 et 0 ", respectivement immobiles dans les référentiels $R$ et $R$ '. Soit A un événement repéré dans $R$ par les coordonnées spatio-temporelles $(x, y, z, t)$. Les coordonnées ( $x^{\prime}, y^{\prime}, z^{\prime}$, t') de ce même événement dans R' (d'axes parallèles à ceux du repère utilisé dans $R$ ) sont données par la transformation suivante (appelée "transformée de Lorentz") :

$$
\text { (1) }\left\{\begin{array}{c}
x^{\prime}=\gamma(x-v t) \\
y^{\prime}=y \\
z^{\prime}=z \\
t^{\prime}=\gamma\left(t-\frac{v}{c^{2}} x\right)
\end{array}\right\} \text { où } \gamma=\frac{1}{\sqrt{1-\frac{v^{2}}{c^{2}}}}
$$

$\gamma$ est appelé "facteur de Lorentz" et est un nombre sans dimension toujours supérieur à 1 , tendant vers l'infini lorsque $v$ tend vers $c$.

Dans cette expression, $v$ représente la vitesse de 0 ' par rapport à 0 , et $c$ représente la vitesse de la lumière; et l'on a choisi les origines spatiales et temporelles dans $R$ et R' de telle sorte que les origines des deux repères coïncident à l'instant $t=0$ et $t^{\prime}=0$. La relativité restreinte part du postulat que la vitesse de la lumière est identique dans $R$ et dans R'.

L'expression (1) montre clairement que deux événements ayant la même coordonnée temporelle, $t$, dans le référentiel $R$ (c'est-à-dire deux événements simultanés dans $\mathrm{R})$ n'ont généralement pas la même coordonnée temporelle, $t$ ', dans le référentiel $R$ ', et n'y sont donc pas simultanés. Il suffit pour cela qu'ils aient des coordonnées $x$ différentes. Cette propriété rend délicate la définition de la longueur d'un objet en mouvement. Dans son référentiel propre, un corps est par définition toujours au repos, et sa longueur (dans la direction $\mathrm{x}$ ) est définie comme la différence des coordonnées de position repérant ses deux extrémités, qui ne varient pas au cours du temps. Par exemple, la longueur propre d'un corps immobile dans le référentiel R' s'écrit $L_{0}=\Delta \mathrm{X}$

En revanche, dans un référentiel où ces coordonnées varient, parce que le corps y est en mouvement, il convient de préciser à quel instant on les considère. La longueur de ce corps ne peut être définie que comme la différence des coordonnées de position repérant ses extrémités, considérées au même instant dans le référentiel en question (c'est-à-dire, en général, à des instants différents dans le référentiel propre dudit corps). Dans l'exemple précédent, on aura alors, pour la longueur $L$ du corps dans le référentiel $\mathrm{R}$, l'expression $\mathrm{L}=\Delta \mathrm{X}$ où les coordonnées $x$ des deux extrémités doivent correspondre à deux événements de même coordonnée temporelle $t$.

Le calcul, immédiat à partir de l'expression (1), conduit à la formule $L=L_{0} / \gamma$, qui 
indique que la longueur d'un corps dans un référentiel où il est en mouvement est toujours inférieure à sa longueur propre. La contraction des longueurs est la conséquence d'un changement de référentiel relativiste.

En réalité, cependant, ce phénomène de "contraction des longueurs", qui est intrinsèquement lié à la physique relativiste, n'est pas directement visualisable per se. En effet, si un observateur est présent dans une scène où se déplacent des objets à une vitesse proche de $c$, il verra certes les objets déformés, mais cette déformation n'aura pas pour unique origine l'effet " minkovskien " indiqué ci-dessus. Ce dernier sera conjugué à un effet lié à la prise en compte de la durée mise par la lumière pour parcourir la distance entre le point de l'objet d'où elle a été émise et l'œil de l'observateur - prise en compte nécessaire puisque la vitesse de $c$ ne peut plus être considérée comme infinie.

\subsubsection{Effets lié à la prise en compte du caractère fini}

\section{de la vitesse de la lumière}

Pour comprendre ces effets, il est nécessaire de concevoir que les photons qui atteignent l'œil de l'observateur à un instant t donné peuvent avoir été envoyés par différents points de la scène, à des instants différents : les photons provenant d'objets proches de l'observateur auront par exemple été émis plus tard que les photons émis par des objets plus éloignés de l'observateur. Ceci est sans conséquence tant que l'observateur et les objets de la scène sont immobiles les uns par rapport aux autres: dans la mesure où chaque point des objets émettant de la lumière se trouve toujours au même endroit, relativement à l'observateur, peu importe l'instant d'émission des photons que celui-ci perçoit, c'est toujours du même lieu qu'il les voit provenir. En revanche, il en va autrement lorsque les objets de la scène se mettent à bouger par rapport à l'observateur. Le lieu où il perçoit chacun des points d'un objet à un instant donné n'est pas le lieu où ils se trouvent maintenant, mais le lieu où ils se trouvaient à l'instant où les photons qu'il perçoit maintenant ont été émis. Or cet instant, forcément antérieur à l'instant d'observation, est d'autant plus reculé dans le passé que la distance à parcourir entre le point en question et l'observateur est grande. La prise en compte simultanée de ce " retard à la perception " et du mouvement des objets (qui fait que la position occupée par un point de l'objet dépend de l'instant considéré) conduit à une déformation apparente des objets. Dans le cas de la métaphore du billard, il est aisé de prévoir - et on l'observe sans équivoque sur les images simulées - qu'un palet s'approchant de l'observateur paraît étiré dans la direction du mouvement, tandis qu'un palet s'éloignant de l'observateur paraît comprimé dans cette même direction (les autres directions n'étant pas affectées). Un autre effet notable découle de la même combinaison de mouvement et de retard à la perception : un observateur immobile par rapport à la table verra les palets se déplacer apparemment plus vite (respectivement moins vite) s'ils viennent vers lui (respectivement s'ils s'éloignent de lui) qu'ils ne le font en réalité : la vitesse apparente est différente de la vitesse relative des objets par rapport à l'observateur. 
Un graphique d'espace-temps nous permet d'expliciter l'origine de cette déformation, et de la quantifier (figure 2).

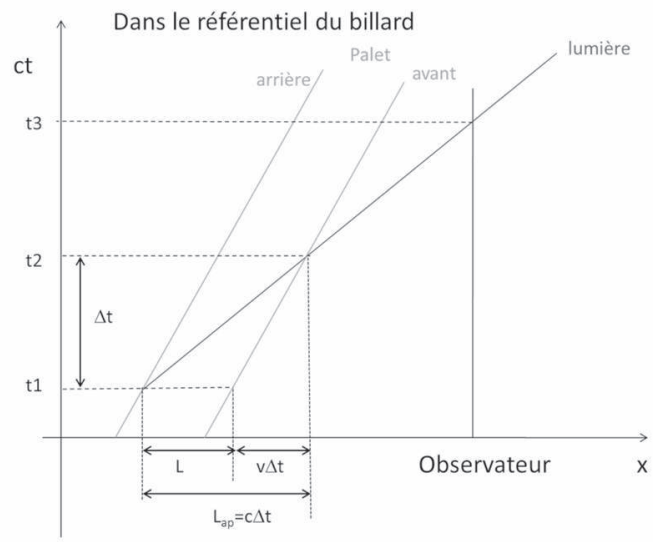

Fig. 2 : mise en graphique du mouvement d'un palet de billard sur une table et dirigé vers un observateur immobile par rapport à la table Sur le graphique, la vitesse du palet (inverse de la pente des droites rouges) et la vitesse de la lumière (inverse de la pente de la droite bleue) sont ramenées à des valeurs proches.

La lecture de ce graphique montre que l'observateur (dont la ligne d'univers est représenté par une ligne verticale, à une abscisse $x$ constante) reçoit à l'instant $t_{3}$ la lumière émise par l'avant du palet à un instant antérieur $t_{2}$, et par l'arrière du palet à un instant encore antérieur $t_{1}$. La longueur du palet, telle que perçue par l'observateur en cet instant $t_{3}$, est donc égale à la différence entre les abscisses de l'avant et de l'arrière du palet, chacun à l'instant qui le concerne. Cette longueur est notée $L_{a p}$ sur le graphique, et l'on voit qu'elle n'est rien d'autre que la distance parcourue par la lumière entre les instants $t_{1}$ et $t_{2}$, soit c $\left(t-t_{1}\right)$. Sur le graphique, le palet se rapproche de l'observateur, et l'on voit que sa longueur apparente est la somme de sa longueur instantanée dans $\mathrm{R}$ notée ici $L$, et de la distance parcourue par le palet (à sa propre vitesse $v$ ) pendant le même intervalle de temps, soit $v\left(t-t_{1}\right)$. Comme nous l'avons déjà vu, la longueur instantanée du palet dans R n'est pas la longueur propre $L_{0}$, mais cette longueur " contractée " d'un facteur $\gamma: L=L_{0} / \gamma$. Un calcul simple donne alors la longueur apparente :

$$
L_{a p}=L_{0} \sqrt{(1+\beta) /(1-\beta)} \text { où } \beta=v / c
$$

Formule 2

De fait, la longueur apparente des palets s'approchant de l'observateur $(0<\beta<1)$ est supérieure non seulement à leur longueur " réelle» dans son référentiel (qui est 
$\left.L=L_{0} / \gamma\right)$, mais également à leur longueur propre, $L_{0}$ Cette longueur apparente tend même vers l'infini lorsque la vitesse relative d'approche du palet tend vers la vitesse de la lumière (c'est-à-dire lorsque $\beta$ tend vers 1 ). Un raisonnement en tout point analogue montre que la longueur apparente d'un palet s'éloignant de l'observateur est au contraire plus courte que sa longueur propre, et même que sa longueur contractée. C'est en fait la même formule qui s'applique, mais avec cette fois - $1<\beta<0$. La longueur apparente tend vers zéro lorsque la vitesse d'éloignement tend vers $c$ (c'est-à-dire tend vers -1).

Dans un monde où toutes les vitesses de déplacement sont ramenées à des valeurs proches de celle de la lumière, les objets en mouvement sont perçus déformés, tantôt allongés, tantôt raccourcis. À l'effet minkovskien de contraction des longueurs se conjugue celui lié à la prise en compte de la durée mise par la lumière pour parcourir la distance qui sépare le point d'émission de l'œil de l'observateur. L'association de ces deux effets rend impossible la visualisation de la contraction des longueurs seule, à moins de faire en sorte que la lumière ne se propage plus depuis les objets en mouvement. On ne peut donc pas attendre d'une simulation de scènes relativistes qu'elle donne directement accès aux effets minkovskiens; on peut en revanche espérer qu'elle permette la mise en œuvre de raisonnements appuyés sur le rôle de la lumière dans la vision.

Plusieurs équipes de chercheurs ont utilisé la simulation immersive (Weiskopf et al., 2006) et la simulation vidéo pour mettre en image des scènes relativistes (Adams et al., 2006; Savage, Searle \& McCalman, 2007; Ruder et al., 2008). Savage et ses collaborateurs (2007) ont testé l'impact d'un simulateur vidéo (non immersif) de voyage relativiste avec des étudiants australiens de première année d'université ayant préalablement reçu un enseignement théorique élémentaire de physique relativiste (aspects cinématiques et optiques). Les étudiants étaient laissés libres d'augmenter ou de diminuer la vitesse et la direction d'un véhicule à bord duquel ils se trouvaient virtuellement (sur lequel était placée la caméra qui filmait la scène qui se déroulait sur l'écran). L'augmentation de la vitesse (jusqu'à des valeurs proches de celle de la lumière, $c$ ) faisait apparaître des effets inattendus (modification des couleurs, déformation des objets de la scène). Savage et son équipe ont montré que l'usage libre du simulateur n'avait eu que très peu d'impact sur la compréhension des phénomènes visualisés (phénomènes d'aberration, effet Doppler relativiste); de nombreux étudiants semblaient se perdre dans des observations dont ils soulignaient le caractère surprenant (qu'ils attribuaient parfois à un "bug" de la programmation) et qu'ils ne cherchaient pas à expliquer.

EVEILS se distingue des simulateurs antérieurs à plusieurs titres, à la fois technologiques et didactiques. D'abord, dans les scènes programmées par l'équipe EVEILS, plusieurs objets peuvent être mis en mouvement les uns par rapport aux autres, et l'observateur lui-même est libre de se mettre en mouvement par rapport aux objets de la scène. Jusqu'ici, les simulateurs permettaient seulement qu'un observateur soit en mouvement par rapport à une série d'objets immobiles les uns 
par rapport aux autres (ou bien qu'un objet se meuve par rapport à une scène dans laquelle l'observateur était lui-même immobile). Ensuite, les scènes et la métaphore du billard français ont été choisies et programmées en référence à des difficultés de compréhension identifiées préalablement par les chercheurs en didactique de l'équipe EVEILS (de Hosson, Kermen \& Parizot, 2010), difficultés que nous rappellerons plus loin. Le contenu et l'organisation des scènes d'immersion devaient favoriser le dépassement de ces difficultés; en outre, les expériences australiennes (Savage, Searle \& McCalman, 2007) et nord-américaines (Adams et al., 2006) nous ont convaincus de guider l'immersion par des suggestions de déplacements, des jeux de questions/ réponses portant notamment sur les différents effets visualisés (de Hosson et al.,2012).

\subsection{De la visualisation immersive à la visualisation vidéo}

Entre 2011 et 2012, nous avons examiné la façon dont cinq utilisateurs de la CAVE s'appropriaient l'environnement métaphorique qui leur était proposé (Maisch, de Hosson \& Kermen, 2012). Nous avons également cherché à identifier les éléments qui leur permettaient de comprendre les effets qu'ils percevaient. Nous nous sommes attachés pour cela au choix de leurs mouvements et de leurs gestes, aux moments où ils décidaient de "geler" une scène ${ }^{3}$ et aux raisons qui les conduisaient à le faire, au contenu de leurs commentaires, etc. Le faible échantillon d'utilisateurs ne permet aucune forme de généralisation, mais nous avons pu remarquer que certains mouvements (se baisser, se lever, face à un palet avançant et ou reculant, suivre un palet à la même vitesse que celui-ci), certaines positions (face à deux palets avançant et reculant et à égale distance de ceux-ci, ou face à l'un des deux) répétés plusieurs fois au fil de l'expérimentation favorisaient l'expression de raisonnements tenant compte du délai de propagation de la lumière entre les instants d'émission et de perception. Au début de l'immersion, les déformations étaient attribuées aux seuls effets "minkovskiens", effets qui, pourtant, ne peuvent pas être visualisés seuls.

Malheureusement, un environnement immersif tel que celui que nous avons utilisé au long du projet EVEILS n'est pas utilisable dans un contexte usuel d'apprentissage (en classe et/ou en formation d'enseignants). Aussi, afin de profiter des potentialités de la visualisation 3D de scènes relativistes, nous avons choisi d'extraire six séquences du protocole d'immersion, testées préalablement avec les cinq utilisateurs de la CAVE, et de les enregistrer en format vidéo. Une telle adaptation contraint fortement les potentialités que nous avons exposées précédemment. En particulier, les mouvements et positions au sein des différentes scènes ne sont plus laissés au libre choix de l'utilisateur; ce dernier est fixe devant un écran sur lequel il voit des déplacements qui ont été choisis en dehors de lui mais avec des intentions didactiques précises (dont l'intérêt a été inféré à partir de la recherche conduite en

3 À tout moment dans la CAVE, les utilisateurs pouvaient stopper le déroulement du jeu de billard, et se déplacer librement pour observer les positions et formes respectives des objets, la simulation étant alors arrêtée et les mouvements de l'utilisateur non traqués. 
immersion). Notre expérimentation inclut donc un référent empirique constitué de six vidéos jouant six scènes supposées "porteuses de sens" par les chercheurs. La manipulation du référent empirique est contrainte et limitée : les utilisateurs peuvent rejouer la scène, faire un arrêt sur image, avancer, reculer le déroulement de la bande-vidéo. L'usage de la vidéo est, pour chacune des scènes, accompagné d'un schéma indiquant la position de la caméra qui filme la scène (c'est-à-dire celle de l'utilisateur) par rapport à la table de billard et aux palets.

\section{Objectifs de la recherche}

Notre travail vise à enrichir les recherches conduites dans l'intention d'évaluer l'impact de l'usage de simulations pour l'apprentissage, celui de la physique en particulier. En 2003, la revue Didaskalia publie un ensemble de travaux consacrés aux liens entre environnements informatiques et enseignement de la physique. Beaufils et Richoux (2003) y présentent un état des lieux de l'usage des simulations en contexte scolaire et proposent un schéma théorique permettant de caractériser les activités associées à l'usage des simulations. La simulation y est définie comme la mise en œuvre de méthodes numériques de traitement d'équations dont les solutions analytiques se trouvent hors de portée des utilisateurs. Dans sa dimension prospective, "la simulation vise la découverte ou la production d'un résultat non évident" (Beaufils \& Richoux, 2003 , p. 19) et se présente comme un plan intermédiaire entre le monde matériel et le monde des théories et des modèles en prenant comme référent soit la phénoménologie, soit le modèle. Dans notre travail, la simulation de scènes relativistes s'ancre dans une phénoménologie "non évidente", c'est-à-dire le monde tel qu'il serait perçu si toutes les vitesses de déplacement étaient ramenées à des valeurs proches de celle de la lumière; et les tâches associées à son usage, relevant essentiellement de l'observation, visent en premier lieu à " renforcer des connaissances factuelles et des aptitudes à la reconnaissance de phénomènes " (ibid.). La construction de savoirs nouveaux (notamment relativistes) paraît plus difficile dans la mesure où la visualisation est le résultat d'une conjugaison d'effets. Outre la question de la nature et de la visée des simulations, se pose également celle des usages. Dans ce contexte, Mellar et Bliss (1994) soulignent que selon les activités et les modes d'apprentissages qu'elles favorisent, les simulations peuvent être de deux types : le premier associe simulation et activités "d'exploration", le second, simulation et activité de "construction". La simulation que nous utilisons dans cette recherche relève du premier type dans la mesure où elle ne permet pas de réelle interaction (manipulation) avec les objets simulés dans le but, par exemple, de construire des modèles explicatifs et/ou prédictifs. Il s'agit ici de donner à voir ce qui n'est pas accessible à la perception ordinaire, en proposant la visualisation d'un phénomène par changement d'échelle (ici de vitesse). Ces visualisations par changement d'échelle sont à l'œuvre dans plusieurs travaux de recherche en didactique des sciences (Buty, 2002; Vince \& Tiberghien, 2000 ; Richoux, Salvetat \& Beaufils, 2002 ; Chauvet \& Duprez, 2003; Finkelstein et al., 
2005) mais, en dehors des travaux auxquels nous avons d'ores et déjà fait référence (Savage, Searle \& McCalman, 2007; Ruder et al., 2008), la plupart d'entre eux engagent des changements d'échelle de longueur. À titre d'exemple, la plateforme conçue par Chauvet et son équipe permet aux utilisateurs d'avoir accès aux conséquences de la théorie cinétique des gaz en observant le mouvement d'une paroi mobile soumise, de part et d'autre, à différents mouvements et chocs particulaires (Chauvet \& Duprez, 2003); celle présentée par Finkelstein et ses collaborateurs (2005) donne à voir le mouvement des électrons au sein de différents éléments de circuits afin d'approcher les lois de l'électrocinétique élémentaire. Dans tous les cas, les recherches conduites à partir de ces simulateurs valorisent leur utilisation pour la compréhension des savoirs (lois, concepts) engagés. Précisons, en outre, que l'ensemble de ces recherches présente la particularité d'adresser chaque fois des difficultés cognitives précises et préalablement identifiées par les concepteurs des simulations eux-mêmes. C'est une démarche analogue qui nous conduit ici à scénariser nos simulations en référence aux difficultés associées à la compréhension des effets relativistes.

\subsection{Les difficultés des étudiants en relativité restreinte}

Les études menées pour caractériser les difficultés des étudiants à propos de la relativité restreinte ne sont pas nombreuses. Néanmoins à partir de ce qui a été exploré, nous pouvons retenir que les étudiants appliquent des lignes de raisonnement "spontané" détectées en cinématique classique (telles que le fait de considérer le mouvement, les distances et les vitesses comme absolus) à des situations construites dans un cadre relativiste (Saltiel \& Malgrange, 1980; Villani \& Pacca, 1987). Ils associent l'ordre selon lequel deux événements sont produits à l'ordre selon lequel ces événements sont perçus. Autrement dit, l'énoncé "deux événements $A$ et $B$ sont perçus de manière successive (A avant B)" est directement associé à l'énoncé "L'événement A s'est produit avant l'événement B", même lorsque les étudiants disposent des informations nécessaires à la résolution du problème (distance, temps de parcours de l'information visuelle, etc.) (Scherr, Schaffer \& Vokos, 2001; de Hosson, Kermen \& Parizot, 2010). Il a ainsi été montré que deux événements simultanés pour un observateur pouvaient être considérés par la plupart des étudiants comme non simultanés par un autre observateur y compris lorsque ces deux observateurs sont immobiles l'un par rapport à l'autre - ici, c'est le caractère opératoire du concept de "référentiel " qui fait défaut (de Hosson, Kermen \& Parizot, 2010). En fait, les étudiants ont des difficultés à reconstruire l'" histoire" d'un photon émis par un objet éclairé, c'est-à-dire à localiser l'événement "émission d'un photon " dans l'espace et dans le temps à partir de l'événement "perception de ce photon " par un observateur, car ils ne prennent pas en compte la distance parcourue par les photons y compris lorsque celle-ci est explicite. Dans certains cas, la propagation de la lumière entre l'objet et l'œil de l'observateur semble même inexistante ou non nécessaire pour expliquer la perception d'un objet par un observateur, ce qui n'est pas sans rappeler les difficultés des élèves associées à la vision des objets (Guesne, 1984). 


\subsection{Problématique et questions de recherche}

Le choix des six scènes a été conditionné par la connaissance que nous avions des difficultés précédentes et par les premiers résultats de l'enquête conduite en immersion. L'objectif de cette étude est de mesurer les apports didactiques d'une visualisation d'effets inaccessibles à la perception ordinaire. Spécifiquement, il s'agit d'étudier l'impact de six vidéos 2D dans lesquelles des scènes 3D relativistes ont été enregistrées, sur la compréhension des conséquences d'un rapprochement des vitesses de déplacement d'objets macroscopiques de celle de la lumière. Il s'agit en particulier de permettre aux utilisateurs potentiels d'approcher la notion d'événement nécessaire à la compréhension de certains aspects de la cinématique relativiste en leur donnant la possibilité de reconstruire, à partir de l'information visuelle reçue par l'œil, le chemin parcouru par la lumière depuis un point d'un objet (localisé dans l'espace et le temps) jusqu'à leur œil, et de remonter ainsi à la localisation spatio-temporelle des événements "émission d'un photon ". Les images perçues par les utilisateurs des vidéos ne peuvent être interprétées qu'en acceptant que chaque point des objets perçus soit une source de lumière, ce qui revient à définir l'émission d'un photon comme un "événement " localisé dans l'espace et dans le temps. Nous faisons l'hypothèse qu'en créant des situations nécessitant, pour être comprises, la mise en place de raisonnements sur les coordonnées spatio-temporelles des événements "émission d'un photon", nous allons permettre aux utilisateurs des vidéos d'accéder ultérieurement aux effets relativistes tels que la non-simultanéité. En effet, comprendre que la simultanéité de deux événements dépend du référentiel d'étude nécessite de localiser les événements non seulement dans l'espace (ce qui est suffisant en cinématique classique) mais également dans le temps.

Les situations choisies reposent sur des choix dont nous souhaitons éprouver la pertinence. En particulier, nous nous demandons quels sont les éléments conceptuels accessibles par le biais des vidéos. Les réponses à cette question engagent deux dimensions:

- Une dimension ergonomique : a-t-on bien choisi les angles de vues/les déplacements des observateurs?

- Une dimension didactique : les effets visibles mis en scène permettent-ils la mobilisation d'éléments conceptuels relevant de la physique de la lumière et de la relativité restreinte?

\section{Méthodologie de recueil et d'analyse des données}

\subsection{Protocole d'expérimentation}

Les six simulations vidéo prévoient l'observation d'une scène dans laquelle des palets se déplacent sans frottement sur une table de billard ${ }^{4}$. Si l'on excepte la première

4 Les collisions des palets avec les bords de la table de billard s'effectuent également sans frottement. 
simulation (où la vitesse de la lumière est laissée à $300000 \mathrm{~km} / \mathrm{s}$ ), la lumière se déplace à une vitesse proche de celles des palets et de l'observateur $(c=1 \mathrm{~m} / \mathrm{s})$. Les six scènes ont été choisies et tournées de façon à favoriser une conceptualisation progressive : même si tous les effets sont visibles en même temps, l'attention de l'utilisateur est concentrée sur l'un ou l'autre de ces effets si bien qu'à chaque vidéo est associée une tâche d'observation pour l'utilisateur (voir tableau 1) guidée par le chercheur/ intervieweur. De nombreux effets sont observables à chaque fois mais l'attention de l'étudiant est portée sur l'un d'eux de façon à permettre une appropriation progressive des éléments conceptuels engagés. L'annexe 1 présente, pour chacune des vidéos, des captures d'écran décrites selon la position et les mouvements de la caméra.

\subsection{Déroulement de l'expérimentation}

Notre expérimentation prend la forme d'entretiens individuels instrumentés d'une demi-heure auprès de neuf étudiants de première année de master enseignement et formation physique-chimie (voir tableau 2). Chaque étudiant, face à un ordinateur, a pour tâche d'observer les scènes jouées dans la simulation et de les commenter à voix haute en présence du chercheur-intervieweur. Le visionnage des vidéos est soutenu par un guidage faible de l'intervieweur, son rôle se limitant à quelques interventions de relance et de demande d'explicitation. Est mesurée ici la capacité des vidéos à provoquer des explications scientifiquement acceptables à partir de l'interprétation des phénomènes observés. À cette approche, que nous pourrions qualifier de constructiviste, nous associons une méthode de recueil de données autorisant l'interviewé à énoncer ses pensées au fur et à mesure qu'il entre en contact avec le matériel que l'on souhaite expérimenter : la méthode de la pensée à voix haute. Celle-ci nous permet d'élucider l'heuristique utilisée par les étudiants pour expliquer les phénomènes observés dans les vidéos (Van Someren, Barnard \& Sandberg , 1994).

Plus précisément, le déroulement d'un entretien prend la forme suivante : avant de présenter l'objectif de l'entretien, le chercheur pose une première question à l'étudiant: "Pouvez-vous expliquer comment on voit les objets qui nous entoure?". Puis il indique que durant l'entretien l'étudiant devra expliquer ce qu'il voit sur les vidéos qui lui sont présentées, sauf pour la première. La passation de la première vidéo permet de montrer comment il est possible de manipuler le référent empirique (pause, explication des prises de vue en travelling), afin d'observer l'aspect des deux palets qui se déplacent à une vitesse totalement négligeable devant celle de la lumière, ce qui est habituel. Il s'agit d'esquisser une première familiarisation avec le rendu de la simulation mais sans latitude laissée à l'étudiant pour prendre en main l'ordinateur.

À la suite de cette première vidéo, le chercheur précise que la vitesse de la lumière valait $300000 \mathrm{~km} / \mathrm{s}$ et que "dans les prochaines vidéos, cette vitesse va être fixée à $1 \mathrm{~m} / \mathrm{s}$ et va avoir pour caractéristique d'être constante et finie". Une deuxième question est alors posée aux étudiants : "Que signifie le fait que la lumière soit finie et constante, pour vous?". Ces questions préalables ont pour but de rendre disponibles (qu'ils se 


\begin{tabular}{|c|c|c|c|}
\hline Vidéo & Durée & Effets visualisés & Objectifs didactiques \\
\hline 1 & $1 \mathrm{~min} 00 \mathrm{~s}$ & $\begin{array}{l}\text { Des palets non déformés se } \\
\text { déplacent de manière conforme à ce } \\
\text { qui pourrait être attendu dans la vie } \\
\text { ordinaire. }\end{array}$ & $\begin{array}{l}\text { Appropriation/familiarisation avec le } \\
\text { principe des simulations-vidéo : place } \\
\text { de la caméra, éléments dynamiques } \\
\text { (pas de frottements), possibilités } \\
\text { ergonomiques, etc. }\end{array}$ \\
\hline 2 & $0 \mathrm{~min} 32 \mathrm{~s}$ & $\begin{array}{l}\text { Les palets frappent la table non } \\
\text { simultanément. Ils sont déformés } \\
\text { (inclinés dans le sens de leur } \\
\text { mouvement), ils accélèrent lorsqu'ils } \\
\text { avancent vers la caméra, décélèrent } \\
\text { lorsqu'ils s'en éloignent. }\end{array}$ & $\begin{array}{l}\text { Construire une explication permettant } \\
\text { de comprendre pourquoi la collision des } \\
\text { palets avec la table n'est pas simultanée } \\
\text { alors que les palets se sont mis en } \\
\text { mouvement au même instant, qu'ils } \\
\text { avancent à la même vitesse par rapport } \\
\text { à la table et qu'ils parcourent une } \\
\text { distance identique. }\end{array}$ \\
\hline 3 & $1 \min 09 \mathrm{~s}$ & $\begin{array}{l}\text { Les palets sont déformés (inclinés } \\
\text { dans le sens de leur mouvement), ils } \\
\text { accélèrent lorsqu'ils avancent vers } \\
\text { la caméra, décélèrent lorsqu'ils s'en } \\
\text { éloignent. }\end{array}$ & $\begin{array}{l}\text { Construire une explication permettant } \\
\text { de comprendre la raison pour laquelle } \\
\text { les palets ont une vitesse plus grande } \\
\text { lorsqu'ils se déplacent vers la caméra, et } \\
\text { plus petite lorsqu'ils s'en éloignent. }\end{array}$ \\
\hline 4 & $1 \min 37 \mathrm{~s}$ & $\begin{array}{l}\text { Les palets subissent des } \\
\text { transformations de forme qui } \\
\text { semblent dépendre de la position } \\
\text { de la caméra : les palets sont étirés } \\
\text { lorsqu'ils avancent vers la caméra, } \\
\text { contractés lorsqu'ils s'en éloignent. }\end{array}$ & $\begin{array}{l}\text { Construire une explication permettant } \\
\text { de comprendre la raison pour laquelle } \\
\text { les palets sont étirés lorsqu'ils avancent } \\
\text { vers la caméra, contractés lorsqu'ils s'en } \\
\text { éloignent. }\end{array}$ \\
\hline 5 & $1 \min 28 \mathrm{~s}$ & $\begin{array}{l}\text { Les palets subissent des } \\
\text { transformations de forme qui } \\
\text { semblent dépendre de la position de } \\
\text { la caméra : les palets sont inclinés. }\end{array}$ & $\begin{array}{l}\text { Construire une explication permettant } \\
\text { de comprendre la raison pour laquelle } \\
\text { les palets s'inclinent. }\end{array}$ \\
\hline 6 & $0 \mathrm{~min} 54 \mathrm{~s}$ & $\begin{array}{l}\text { Des palets fantômes apparaissent } \\
\text { dans la scène. Ils ne subissent pas } \\
\text { les mêmes déformations de forme, } \\
\text { ni de vitesse que les palets "réels" } \\
\text { dont ils sont l'empreinte. }\end{array}$ & $\begin{array}{l}\text { Construire une explication permettant } \\
\text { de comprendre la raison pour laquelle } \\
\text { les palets "fantômes" diffèrent des } \\
\text { palets "réels"; distinguer " effet } \\
\text { minkovskien" et "effets de prise en } \\
\text { compte du caractère fini de c.". }\end{array}$ \\
\hline
\end{tabular}

Tableau 1 : les 6 vidéos de l'expérimentation et les objectifs didactiques associés

Les palets fantômes représentent les palets "réels" lorsque la propagation de la lumière n'est pas prise en compte dans l'observation des objets. Ces palets "fantômes" se déplacent donc à une vitesse de $\mathrm{c}=1 \mathrm{~m} / \mathrm{s}$ mais ne font pas apparaître les modifications dues à la propagation de la lumière. Ils ne visualisent que les effets de contraction des longueurs. Nous avons découplé les effets dus à la prise en compte du caractère fini de la vitesse de la lumière des effets dits "minkovskiens".

\begin{tabular}{|l|c|c|c|c|c|c|c|c|c|}
\hline Étudiant & AB & YS & RG & JT & KdF & JAD & SP & PEMS & SL \\
\hline $\begin{array}{l}\text { Suivi d'un enseignement de relativité restreinte } \\
\text { lors de ses études }\end{array}$ & oui & $?$ & $?$ & non & non & non & oui & oui & oui \\
\hline
\end{tabular}

Tableau 2 : les étudiants interrogés et l'étude de la relativité restreinte 
les remémorent) leurs connaissances sur ce sujet de façon à ce qu'ils les mettent en œuvre plus aisément par la suite, si elles sont opératoires. Après la réponse fournie par l'étudiant, la vidéo 2 est décrite avant d'être visionnée.

Pour chacune des vidéos (2 à 6), la méthodologie est la même : le chercheur décrit la vidéo en précisant, à l'aide d'un schéma (voir annexe 1), le point de départ des palets et leur nombre, le sens de leur mouvement (il est précisé qu'ils ont la même vitesse constante par rapport à la table et qu'ils partent au même instant), la position initiale de l'observateur, puis ses déplacements ultérieurs avant de demander à l'étudiant de décrire ce qu'il voit et de fournir une explication physique des phénomènes visualisés. À chaque passation de vidéo, une consigne précise est fournie, le chercheur invite l'étudiant à revoir la vidéo s'il le souhaite, à faire des pauses. Il veille à ce que l'étudiant parle bien à voix haute durant tout l'entretien et pose des questions d'approfondissement pour vérifier le degré de réflexion de l'étudiant lors de certaines réponses parfois inattendues; aucun élément conceptuel n'est fourni à l'étudiant, à aucun moment de l'entretien.

\subsection{Méthode d'analyse}

Chaque entretien a été enregistré sous format vidéo, puis retranscrit dans le logiciel ELAN. Les réponses aux questions posées après la passation de la première vidéo ont donné lieu à une analyse lexicale.

Puis, à l'aide du logiciel, nous avons effectué un comptage des utilisations du référent empirique et repéré les différentes déclarations dans le temps, pour déterminer devant quelle vidéo les phénomènes ont été décrits ou expliqués. Nous avons d'abord procédé à un repérage, dans les productions verbales des étudiants, des effets visibles mentionnés pour chaque vidéo, puis nous avons catégorisé ces productions verbales en fonction des éléments conceptuels mobilisés (voir tableau 3), de manière à mesurer la pertinence et la richesse des explications fournies. Ensuite, nous avons analysé l'évolution de l'organisation de ces éléments conceptuels au fil des vidéos et pour un même étudiant, autrement dit, la manière dont les différents éléments conceptuels s'agrègent et s'articulent au fil du visionnage. En complément, nous avons mis les productions verbales en relation avec les différents types de manipulation permis par la simulation (mise en pause, relance).

En particulier, nous nous attachons à repérer les éléments conceptuels mentionnés dans le tableau 3.

\begin{tabular}{|l|l|}
\hline 1 & L'entrée de la lumière dans les yeux de l'observateur \\
\hline 2 & Le caractère fini de la vitesse de la lumière \\
\hline 3 & La distance entre les objets et l'observateur \\
\hline 4 & L'émission de photons par différents points de l'objet \\
\hline 5 & Les modifications géométriques imputables à la transformée de Lorentz \\
\hline
\end{tabular}

Tableau 3 : les éléments conceptuels impliqués dans les explications des phénomènes observés dans les vidéos de la simulation 
Cependant, à la lecture des entretiens, il est apparu que certains des éléments conceptuels étaient utilisés de manière implicite, ce qui nous a conduits à distinguer les catégories suivantes :

(1i) l'entrée de lumière dans les yeux de l'observateur est suggérée, par exemple "nous apparaît", "on perçoit", dans la mesure où auparavant l'étudiant a mentionné une fois l'entrée de lumière dans les yeux ou encore "on reçoit les rayons ou la lumière" ou toute expression ayant ce sens, ce qui correspond à une reconnaissance implicite de l'entrée de la lumière dans l'œil de l'observateur;

(2i) le caractère fini de la vitesse de la lumière est évoqué de façon implicite dans des déclarations comme "vitesse de la lumière proche de celle des palets" ou "la lumière va plus vite" ou toute expression ayant ce sens. Dans d'autres cas, le caractère fini de la vitesse de la lumière est sous-entendu et ce sont ses conséquences qui sont utilisées : "temps mis par la lumière plus court ou plus long". Nous avons considéré que toute expression ayant ce sens, associée à la mention d'une distance entre l'objet et l'observateur, correspondait à une reconnaissance implicite du caractère fini de la vitesse de la lumière.

(3i) la distance entre l'objet et l'observateur est prise en compte de façon implicite dans des expressions telles que "plus loin ou plus proche" (ou toute expression de ce type), dans la mesure où la position de l'observateur a été mentionnée au préalable;

(4i) différents points ou parties du palet sont pris en compte dans le raisonnement mais l'émission de lumière est sous-entendue.

\section{Résultats}

Dans ce paragraphe, nous présentons les analyses lexicales des réponses aux questions préalables, puis les descriptions des phénomènes perçus par les étudiants, en lien avec l'analyse des explications proposées.

\subsection{Analyse des réponses aux questions préalables}

Concernant l'explication de vision des objets, on attend des étudiants qu'ils mobilisent les éléments de la chaîne de raisonnement proposée par Chauvet (1996) : mention de lumière, diffusion de lumière, trajet de la lumière de l'objet vers les yeux, arrivée de la lumière dans les yeux, formation d'une image dans le cerveau. Trois étudiants ont une certaine difficulté à fournir une réponse complète, SP reste très évasive "Ben, je dirais le fait qu'il soit éclairé par la lumière", RG ne complète pas beaucoup plus "Physiquement? Ben euh, donc il en, il reçoit de la lumière et il la renvoie", ces deux étudiantes s'attachant à la première partie de l'explication tandis que SL s'intéresse plutôt à la dernière partie "Là on voit l'objet, hein. Euh. Donc on a des rayons lumineux qui parviennent jusqu'à notre œeil. Voilà" et ajoute qu'il ne voit pas quoi "dire d'autre". Les autres fournissent une explication complète ou quasi complète. AB ne précise pas d'où provient la lumière mais décompose l'objet vu, "des rayons lumineux 
sont émis dans toutes les directions par chacun des points qui composent les objets". En somme six étudiants sur les neuf arrivent dans cette étape à mobiliser les éléments essentiels qui, selon nous, devraient favoriser la compréhension des simulations qui leur seront ensuite présentées.

À propos du caractère constant de la vitesse de la lumière, six étudiants déclarent qu'il s'agit d'une absence de variation sans plus (SP, PEMS, JAD), dans le temps (RG), quelles que soient les "situations" (KdF, discutable) ou les "milieux" ( $\mathrm{SL}$, à tort). AB dit que c'est un "principe fondamental" sans expliciter davantage et JT annonce qu'elle ne peut dépasser une certaine valeur.

Concernant la valeur finie de la vitesse de la lumière, Kdf affirme que cet adjectif signifie "défini, connu". Au contraire YS s'attache à dire ce que cela signifie pour l'objet qui est regardé, il est vu tel qu'il était un instant plus tôt. L'ensemble des réponses présente une caractéristique commune, aucune d'elles ne rend compte du fait qu'un objet ne peut se déplacer à une vitesse égale ou supérieure à la vitesse de la lumière, quel que soit le référentiel considéré.

\subsection{Analyse des explications fournies par les étudiants}

Les éléments descriptifs utilisés par les étudiants pour identifier les phénomènes soumis à leur observation dans les vidéos 2 à 5 sont rassemblés en annexe. Afin d'avoir une vue d'ensemble, nous avons reporté dans le tableau 4 le numéro de la vidéo durant la passation de laquelle l'étudiant a décrit le phénomène. Le tableau permet de savoir si un phénomène a été décrit par l'étudiant et dans quelle vidéo et de visualiser l'absence de description d'un phénomène par un étudiant.

\begin{tabular}{|l|c|c|c|c|c|c|c|c|c|}
\hline & $\mathbf{A B}$ & YS & RG & JT & KdF & JAD & SP & PEMS & SL \\
\hline décalage & $\mathrm{v} 2, \mathrm{v} 3$ & $\mathrm{v} 2$ & $\mathrm{v} 2, \mathrm{v} 4$ & $\mathrm{v} 2$ & $\mathrm{v} 2, \mathrm{v} 3$ & $\mathrm{v} 2, \mathrm{v} 5$ & & $\mathrm{v} 2$ & $\mathrm{v} 2$ \\
\hline vitesse & $\mathrm{v} 3, \mathrm{v} 4$ & $\mathrm{v} 3$ & & $\mathrm{v} 4$ & $\mathrm{v} 3$ & $\mathrm{v} 3$ & $\mathrm{v} 3, \mathrm{v} 4$ & $\mathrm{v} 3$ & $\mathrm{v} 4$ \\
\hline déformation & $\mathrm{v} 4$ & $\mathrm{v} 4$ & $\mathrm{v} 5$ & $\mathrm{v} 4, \mathrm{v} 5$ & $\mathrm{v} 4$ & $\mathrm{v} 5$ & $\mathrm{v} 5$ & $\mathrm{v} 4$ & \\
\hline inclinaison & $\mathrm{v} 2$ & $\mathrm{v} 2, \mathrm{v} 4, \mathrm{v} 5$ & & $\mathrm{v} 4$ & $\mathrm{v} 4, \mathrm{v} 5$ & $\mathrm{v} 4, \mathrm{v} 5$ & $\mathrm{v} 4$ & $\mathrm{v} 4$ & $\mathrm{v} 5$ \\
\hline
\end{tabular}

Tableau 4 : description d'un phénomène face à telle vidéo

S'agissant des trois premiers phénomènes "décalage", "vitesse " et "déformation", auxquels les vidéos 2, 3 et 4 étaient respectivement dédiées, les étudiants sont plus nombreux à décrire le phénomène qu'à ne pas le percevoir. Pour le phénomène "inclinaison", sept étudiants l'avaient noté avant la vidéo 5 qui lui est consacrée.

Pour la vidéo 6, il est demandé aux six étudiants qui la visionnent de déterminer quel est le nouvel élément qui a été ajouté dans la simulation et ce qu'il représente.

Tous les étudiants remarquent immédiatement les palets floutés qu'ils nomment diversement, fantômes, images, hologrammes. Cinq étudiants disent que les deux palets floutés ont le même mouvement, JT ajoute que cela se vérifie quelle que soit la position de l'observateur et qu'ils sont partis avant les palets colorés. Il note qu'ils 
sont plus contractés que les autres. JAD et PEMS déclarent qu'ils sont aplatis alors que SP dit qu'ils sont plus petits que les deux autres palets et pas déformés. Elle est la seule à n'avoir fait aucune description du mouvement des palets floutés. Il semble donc que l'effet mis en valeur à chaque étape (c'est-à-dire dans chacune des vidéos) est quasiment identifié par les étudiants.

Dans les lignes qui suivent, nous analysons la manière dont les étudiants expliquent les différents phénomènes qu'ils identifient au fil du visionnage.

\subsubsection{Explications du décalage des collisions}

Cela concerne ce qui a d'abord été vu dans la vidéo 2. Lorsque les deux palets touchent la bande, au même instant (mais cela ne peut pas être vu), la lumière provenant du palet le plus éloigné a une distance plus grande à parcourir que celle provenant du palet le plus proche, pour arriver à l'œil de l'observateur et donner l'information "choc du palet avec la bande". Par conséquent elle met plus de temps à arriver et l'observateur a la sensation que le palet éloigné touche la bande après le palet proche.

\begin{tabular}{|l|c|c|c|c|c|c|c|c|c|}
\hline Étudiants & AB & YS & RG & JT & KdF & JAD & SP & PEMS & SL \\
\hline $\begin{array}{l}\text { Explication du } \\
\text { décalage des } \\
\text { collisions }\end{array}$ & oui & oui & non & oui & oui & oui & non & oui & oui \\
\hline Éléments conceptuels & $1 \mathrm{i}, 2 \mathrm{i}, 3 \mathrm{i}$ & $1 \mathrm{i}, 2 \mathrm{i}$ & & $1 \mathrm{i}, 2 \mathrm{i}, 3$ & $2 \mathrm{i}$ & $1 \mathrm{i}, 2 \mathrm{i}, 3$ & & $1,2 \mathrm{i}, 3$ & $1,2 \mathrm{i}, 3$ \\
\hline $\begin{array}{l}\text { fournis devant la } \\
\text { vidéo }\end{array}$ & $\mathrm{v} 2$ & $\mathrm{v} 2$ & $\mathrm{v} 2$ & $\mathrm{v} 2$ & $\mathrm{v} 2$ & $\mathrm{v2}, \mathrm{v3}$ & $\mathrm{v2}$ & $\mathrm{v2}, \mathrm{v3}$ & $\mathrm{v} 2$ \\
\hline
\end{tabular}

Tableau 5 : explications du décalage des collisions

Deux étudiantes RG et SP, ont été incapables de fournir une explication satisfaisante. L'une dit "ça dépend de la position de l'observateur" ou "ça doit être une question d'optique" sans relier ces arguments. L'autre déclare que "c'est peut-être la vitesse de la lumière qui donne cette impression, que le rouge se déplace plus vite" sans avancer d'autres arguments. Au contraire les autres étudiants, à des degrés divers, implicitement ou explicitement, utilisent certains des éléments conceptuels essentiels que nous avons mentionnés, en les articulant plus ou moins aisément. Par exemple YS et JAD précisent en préambule à leur explication que les deux palets à tout moment sont à la même abscisse (sur un axe parallèle à la grande bande du billard), avant de dire que la vidéo rend compte de la perception de l'observateur. Deux étudiants YS et Kdf, ne prennent pas en compte la distance entre l'observateur et chaque palet, mais s'appuient sur le fait que la lumière parcourt la distance entre les deux palets en une durée qui ne peut être négligée.

\subsubsection{Explications des vitesses différentes à l'aller et au retour}

Le phénomène est particulièrement mis en évidence face aux palets dans la vidéo 3 . Arbitrairement, nous nommons vitesse aller celle qui correspond à la vitesse 
du palet lorsqu'il s'éloigne de l'observateur vers la bande du billard située au fond, et vitesse retour lorsque le palet vient vers l'observateur depuis l'autre extrémité du billard, donc se rapproche de lui.

\begin{tabular}{|l|c|c|c|c|c|c|c|c|c|}
\hline Étudiants & AB & YS & RG & JT & KdF & JAD & SP & PEMS & SL \\
\hline $\begin{array}{l}\text { Explication vitesse aller plus } \\
\text { petite }\end{array}$ & 0 & 0 & - & 0 & 0 & 0 & $\mathrm{n}$ & $\mathrm{n}$ & $\mathrm{n}$ \\
\hline $\begin{array}{l}\text { Explication vitesse retour } \\
\text { plus grande }\end{array}$ & 0 & - & - & 0 & 0 & 0 & $\mathrm{n}$ & $\mathrm{n}$ & $\mathrm{n}$ \\
\hline Éléments conceptuels & $1,2 \mathrm{i}, 3,5$ & $1 \mathrm{i}, 2 \mathrm{i}, 3$ & & 2,3 & $2 \mathrm{i}$ & $2 \mathrm{i}, 3$ & & 1,3 & $1,2 \mathrm{i}, 3$ \\
\hline Fournis devant vidéo & $\mathrm{v} 2$ & $\mathrm{v} 3$ & & $\mathrm{v} 4$ & $\mathrm{v3}$ & $\mathrm{v} 3$ & $\mathrm{v3}$ & $\mathrm{v5}$ & $\mathrm{v} 4$ \\
\hline
\end{tabular}

Tableau 6 : explications des vitesses aller et retour différentes - : pas évoqué ; 0 : arguments articulés; $\mathrm{n}$ : arguments non articulés ou incorrects

L'explication attendue, outre le fait de comporter certains des éléments conceptuels essentiels, doit être cohérente pour les deux aspects, vitesse aller, vitesse retour. RG n'ayant pas identifié le phénomène ne l'explique pas. Cependant trois autres étudiants (SP, PEMS, SL) n'y parviennent pas alors qu'ils l'ont identifié. Plus surprenant, PEMS et SL citent des éléments conceptuels pertinents, sans parvenir à les articuler pour donner du sens à leur propos.

Par exemple SL: "Alors, les, les rayons, qui parviennent jusqu'à notre œeil (1), euh, nous parviennent plus vite (2i) d'un objet plus proche (3), / que d'un objet, euh, plus lointain. Donc, euh. / Donc on est plus sensible à la, à la vitesse d'un objet proche plutôt que d'un, euh, objet, euh, lointain". Le chercheur lui demande de préciser ce qu'il entend par sensible, et sa réponse ne permet pas de comprendre "ça va être plus rapide dans notre perception". Et il ne distingue pas dans sa tentative d'explication la vitesse lorsque le palet s'éloigne et lorsqu'il se rapproche.

Cinq étudiants parviennent à articuler différents arguments pour justifier ces vitesses différentes sans aller cependant au bout de leur raisonnement. YS et $A B$ utilisent le fait que le palet avance à une vitesse constante dans le référentiel du billard. Ils divisent la trajectoire du palet en segments égaux parcourus par le palet lors de durées identiques. Selon que l'on considère le trajet aller ou retour, à chacune de ces durées ils ajoutent ou retranchent la durée mise par la lumière pour apporter cette information à l'observateur, et ne concluent pas sur la vitesse.

$\mathrm{KdF}$ procède un peu différemment, il imagine que le palet est un émetteur de flashs et ajoute "comme s'il m'envoyait, euh, une balle, tous des laps de temps réguliers, quoi. Un truc qui justement aille pas trop vite par rapport à lui " indiquant qu'il est bien conscient que la vitesse de la lumière est finie et que la conséquence est importante. "S'il s'éloigne ça veut dire que ça arrive moins vite vers moi./ Et s'il se rapproche, ça veut dire qu'il arrive plus vite. Hummm. Ouais. // ben je pense que si il s'éloigne, euh. // Ça fait le même effet que si, euh, enfin que sa vitesse, euh, réduit, la vitesse de la lumière. / Ça me gêne de dire ça. J'ai toujours appris que c'était une constante [rire]./ 
Euh, donc réduit la vitesse de la lumière, et donc elle prend plus de temps, euh / pour arriver vers moi à même distance que lorsqu'il se rapproche". II occulte la distance parcourue par le palet entre chaque émission de flash dans son explication, alors qu'elle est un élément essentiel, et considère une émission discontinue de l'onde électromagnétique alors que nous sommes ici dans un cadre non quantique ${ }^{5}$.

$\mathrm{KdF}$, JAD et JT, considèrent la distance parcourue par l'information lumineuse entre le palet et l'observateur pour dire que la vitesse aller est plus petite que la vitesse retour, comme s'ils calculaient une vitesse moyenne en fait, et sans considérer la distance parcourue par le palet à intervalles de temps réguliers, ce qui ne permet pas de conclure sur la nature de cette vitesse aller ou retour, constante ou pas. JAD constate que son raisonnement est en contradiction avec l'observation ce que ne relèvent pas les deux autres.

\subsubsection{Explications de la déformation de la face supérieure des palets}

L'attention des étudiants est attirée sur cet aspect lors de la passation de la vidéo 4.

\begin{tabular}{|l|c|c|c|c|c|c|c|c|c|}
\hline Étudiants & AB & YS & RG & JT & KdF & JAD & SP & PEMS & SL \\
\hline $\begin{array}{l}\text { Explication } \\
\text { étirement si } \\
\text { rapprochement }\end{array}$ & 0 & 0 & - & 0 & 0 & $\mathrm{n}$ & - & $\mathrm{n}$ & $\mathrm{n}$ \\
\hline $\begin{array}{l}\text { Explication } \\
\text { contraction à } \\
\text { l'éloignement }\end{array}$ & - & $\mathrm{n}$ & - & $\mathrm{n}$ & - & - & - & - & - \\
\hline $\begin{array}{l}\text { Éléments } \\
\text { conceptuels }\end{array}$ & $\begin{array}{c}3 \mathrm{i}, \\
4 \mathrm{i}, 5\end{array}$ & $\begin{array}{c}2 \mathrm{i}, \\
3,4\end{array}$ & & $\begin{array}{c}2 \mathrm{i}, \\
3,4\end{array}$ & $4 \mathrm{i}$ & $\begin{array}{c}1 \mathrm{i}, 3, \\
4 \mathrm{i}\end{array}$ & & 1 & $2 \mathrm{i}, 4 \mathrm{i}$ \\
\hline $\begin{array}{l}\text { Fournis devant } \\
\text { vidéo }\end{array}$ & $\mathrm{v} 4, \mathrm{v} 5$ & $\mathrm{v} 4$ & & $\mathrm{v} 5, \mathrm{v} 4$ & $\mathrm{v} 5$ & $\mathrm{v} 5$ & & $\mathrm{v} 5$ & $\mathrm{v} 5$ \\
\hline
\end{tabular}

Tableau 7 : explications des déformations des palets

- : pas évoqué; 0 : arguments articulés; $\mathrm{n}$ : arguments non articulés ou incorrects

Six étudiants ( $\mathrm{AB}, \mathrm{YS}, \mathrm{JT}, \mathrm{KdF}$, JAD et SL) distinguent l'avant du palet et l'arrière, ce qui correspond à l'élément conceptuel 4 ou $4 \mathrm{i}$ selon qu'ils mentionnent la lumière ou pas; $A B, Y S$ et KdF sont plus précis : ils parlent d'un point situé à l'avant du palet et d'un situé à l'arrière.

$\mathrm{AB}$ “je j'vous disais que le point, euh, par exemple ce point-là [point d'un palet, sur le schéma] il avait un retard par rapport au point de devant. Mais ça, mais ça c'est parce que ce point derrière, il est plus loin et du coup, ça faisait comme tout à l'heure où, euh, quand on était ici [petite bande], on avait le point qui était le plus loin, il paraissait

5 En version quantique (avec des photons individuels), on peut en effet parler de rythme (ou fréquence) d'émission, et il est vrai que ce rythme de réception sera différent de celui d'émission. Mais cela n'aura aucune incidence sur la forme apparente de l'objet. Le nombre de photons émis ou reçus par seconde est lié à un autre aspect que nous avons négligé : l'intensité lumineuse. Dans nos simulations, que l'on suppose une base classique ou quantique pour la lumière, la question du "rythme" d'émission n'est pas pertinente. 
avec un retard". Elle évoque le raisonnement précédent qui la conduit à parler de retard du point éloigné, mais elle ne convertit pas cela en longueur. Il est intéressant de noter que $\mathrm{AB}$ a d'abord convoqué le cadre relativiste pour tenter d'expliquer, non seulement la déformation observée mais également les effets précédents :

90, 29:06: Vous avez dit : "je veux bien comprendre qu'ils soient déformés". Alors, euh, qu'est-ce que vous comprenez?

91, 29:16 AB: Ben disons que ça va être cohérent avec ce que j'ai appris.

92, 29:20: Ah ben expliquez nous.

93, 29:22 AB : Euh, parce que, euh. / Ah oui, je suis même pas sûre qu'ils soient déformés dans le bon sens. Mais, euh. / Parce que quand un objet se dé-, se déplace avec une vitesse proche de la lumière par rapport à nous, on le perçoit pas avec les mêmes dimensions que ce qu'il a réellement. /

94, 29:40: Et pour quelles raisons?

95, 29:42 AB : Ben comme je vous l'ai dit tout à l'heure, je crois que je sais pas l'expliquer. Enfin, c'est, c'est les transformations de Lorentz, qui conduisent à ces résultats. C'est pour ça que ça me surprend pas que, qui y a, euh, un rétrécissement en largeur ou un allongement. Ni qu'ils aillent plus ou moins vite puisque le temps ne s'écoule pas de la même manière. / Mais alors que celui de derrière soit, soit pas affecté pareil que celui de devant, je vois pas trop quel outil va me permettre de conclure ça.

La référence au changement de cadre (et à ses conséquences possibles) laisse $A B$ dans une impasse, ce qui la conduit à reprendre un raisonnement fondé sur "l'histoire" des photons émis des différents points du palet observé.

D'ailleurs, $A B, Y S$, JT et KdF se réfèrent tous au type d'explication qu'ils ont déjà utilisé en considérant qu'un point situé sur la partie éloignée du palet est en retard par rapport à un point situé sur la partie avant du palet lorsque celui-ci arrive vers l'observateur, sans convertir cela en terme de longueur perçue pour la surface du palet. Pour la déformation observée lorsque le palet s'éloigne de l'observateur, seuls YS et JT l'évoquent sans être plus explicites.

SL s'engage dans une explication comportant des éléments conceptuels pertinents: "Donc la partie qui va, euh, le devant du palet, va, euh, va nous arriver donc plus vite (2i), euh, que la partie, euh, l'arrière du palet et on va avoir une, euh./ Cette déformation va, euh, va être amplifiée euh, avec, euh, la vitesse, euh du palet". Malgré une demande de précision il n'ajoute pas d'autre argument et ne mène pas l'explication à terme.

JAD indique bien que les parties différentes du palet ne sont pas vues au même moment mais ne dit pas en quoi cela conduit à une surface allongée. Il poursuit ensuite en déclarant " toujours du point de vue perception, on aurait une plus grande différence de vitesse entre l'avant et l'arrière du palet jaune, enfin du palet orange, qu'entre l'avant et l'arrière du palet rouge" ce qui lui permet de dire que cela expliquerait l'étirement du palet tout en ajoutant juste après qu'il n'en est pas sûr. Cet argument est erroné dans la mesure où le palet a un mouvement de translation; s'il disait vrai la forme du palet changerait continuellement sur un trajet aller (ou retour). 


\subsubsection{Explication de l'inclinaison des palets}

Le phénomène est en fait visible dès la vidéo 2 et YS a d'ailleurs amorcé une première explication à ce moment-là. Mais cinq étudiants ne tentent pas d'expliquer l'inclinaison des palets, dont $A B$ qui jusque-là avait combiné différents éléments conceptuels pour débuter une explication des autres phénomènes.

\begin{tabular}{|l|c|c|c|c|c|c|c|c|c|}
\hline Étudiants & AB & YS & RG & JT & KdF & JAD & SP & PEMS & SL \\
\hline Explication inclinaison des palets & - & 0 & - & 0 & 0 & 0 & - & - & - \\
\hline Éléments conceptuels & & $2 \mathrm{i}, 3,4$ & & $1 \mathrm{i}, 2 \mathrm{i}, 3,4$ & $2 \mathrm{i}, 3 \mathrm{i}$ & 4 & & & \\
\hline Fournis devant vidéo & & $\mathrm{v} 2, \mathrm{v} 5$ & & $\mathrm{v} 5$ & $\mathrm{v} 4$ & $\mathrm{v} 5$ & & & \\
\hline
\end{tabular}

Tableau 8 : explications de l'inclinaison des palets - : pas évoqué; 0 : arguments articulés; $\mathrm{n}$ : arguments non articulés ou incorrects

Les quatre autres ne sont pas très précis dans leur mise en œuvre des arguments. YS, JT et JAD distinguent le haut et le bas du palet pour dire que selon la distance à l'observateur, la lumière met moins de temps pour venir du haut que du bas, tandis que KdF évoque la durée mise par la lumière pour parcourir la hauteur du palet. Ils ne relient pas cela clairement à une inclinaison du palet par rapport à la verticale, comme si l'explication fournie pour les phénomènes précédents était suffisante; d'ailleurs YS dit que "c'est toujours pareil, une histoire de différences de distances entre un point du palet et l'autre" après s'être embrouillé et ne pas avoir réussi à être cohérent.

\subsubsection{Explications données aux palets floutés}

À la question essentielle de déterminer ce que représentent les palets floutés, quatre des six étudiants (JT, KdF, SP, SL) expriment l'idée qu'ils figurent le mouvement des palets si la vitesse de la lumière avait sa valeur habituelle de $300000 \mathrm{~km} \cdot \mathrm{s}^{-1}$; cet avis, totalement incompatible avec les durées relatives des mouvements des palets colorés et des palets floutés, montre à quel point les étudiants ont été surpris, alors que par exemple JT et KdF avaient jusque-là fait preuve de cohérence. À leur décharge il est important de rappeler qu'aucun n'a suivi d'enseignement de relativité dans ses études, et que $\mathrm{KdF}$ conteste presque aussitôt cette idée qu'il vient d'émettre en indiquant ce qui lui parait ne pas être compatible avec la déformation des palets floutés. Au contraire SP et SL qui ont suivi un tel enseignement, ne relèvent pas la forme des palets floutés, SP affirme même qu'ils ne se déforment pas, tandis que SL évoque de façon assez peu compréhensible la sensibilité de l'œil qui ne serait pas suffisante pour qu'on puisse alors distinguer la différence de date d'arrivée dans l'œil de la lumière (à sa vitesse habituelle) provenant de chaque palet flouté.

PEMS ne propose aucune interprétation de ce que seraient les palets floutés alors qu'il a suivi un enseignement de relativité restreinte.

JAD fait une déclaration un peu surprenante, parlant de "regarder les palets floutés à une vitesse normale", qui se comportent alors comme en mécanique classique, ce 
qui sous-entend que ce qui lui a été présenté avant relève de la cinématique relativiste, qu'il n'a au demeurant pas étudié dans son cursus. Mais il évacue complètement le rôle de la lumière qu'il a pourtant utilisée dans ses explications précédentes, en omettant d'en parler. Et il règle la question de la forme aplatie des palets floutés qu'il a bien notée, en l'attribuant à un effet de perspective, alors qu'il n'a pas évoqué cette raison auparavant.

Dans les situations précédentes, on pouvait dire que plusieurs étudiants avaient compris le rôle que la durée de propagation de la lumière jouait dans une situation où les palets avaient une vitesse proche de celle de la lumière. Ici il s'avère que la situation présentée n’a pas permis de comprendre ce qui était en jeu.

\subsection{Observations, explications et manipulations des vidéos}

Si l'on dénombre le type de manipulation effectuée par étudiant pour chaque vidéo (voir annexe 2) on s'aperçoit que la répartition est plutôt hétérogène. Autrement dit, il ne semble pas qu'une vidéo incite un nombre particulièrement élevé ou faible de manipulations. Par ailleurs, un nombre identique de manipulations effectuées par plusieurs étudiants pour une même vidéo ne recouvre pas des réalités identiques d'observation ou d'explication. Par exemple AB et SP ont regardé six fois la vidéo 2 mais seule la première a identifié le phénomène de décalage des collisions dès la deuxième passation et l'a ensuite expliqué. Dans la vidéo 4, YS a fait neuf mises en pause, afin d'observer pour décrire avec précision les différentes orientations et formes des palets selon la position de l'observateur. KdF et YS en ont fait neuf et onze, car étant surpris par certaines formes, ils se sont attachés à les examiner une à une, et ont ensuite fourni un début d'explication aux déformations. RG en a fait sept, intriguée par les formes et les différences de vitesse selon la position de l'observateur, mais sans parvenir à formuler une description précise des déformations ni a fortiori une explication.

Nous avons donc cherché à identifier les moments et les manipulations (mises en pause, relances, reprises) qui semblaient favorables à l'amorce d'une explication engageant un ou plusieurs des éléments conceptuels du tableau 3. Pour cela, nous avons repéré les manipulations effectuées par les étudiants au moment où leur production verbale porte la trace de l'un ou plusieurs de ces éléments. D'une manière générale, on s'aperçoit qu'une observation déclarée à voix haute s'accompagne d'une mise en pause puis d'une relance de la vidéo depuis le début. La mise en pause marque ce qui retient l'attention; la relance semble indiquer que l'étudiant cherche dans la genèse du mouvement des palets et de celui de l'observateur une cause à l'effet identifié, comme l'illustre ce commentaire de Kdf : "Bon, j'ai vu ce que je voulais voir. Avec les deux palets, le rouge et le orange, qui sont bien censés être partis en même temps, on retrouve le fait qu'on a l'impression que le rouge est toujours en avance sur le orange. Donc là, je pense que c'est la différence de distance entre les deux. Euh, c'est pour ça que j'ai demandé à revoir la vidéo, c'est parce que je l'ai remarquéà la fin, je voulais être sûr que ce soit le cas dès le début". L'instant de la 
mise en pause peut être considéré comme le marqueur d'une observation féconde : "Bon j'ai vu ce que je voulais voir".Ce n'est pas l'observation du décalage avec lequel les deux palets frappent la table qui permet à Kdf de construire une explication (même si celle-ci est incomplète) mais l'observation répétée (cinq fois, dans le cas présent) des collisions mise en perspective avec l'observation des palets au démarrage. De la même manière, ce n'est pas l'observation de la déformation des palets qui s'avère déterminante, mais cette observation mise en lien avec la configuration sous laquelle la déformation disparait. Les relances sont souvent effectuées par les étudiants pour attraper cette mise en lien-là. Ainsi RG trouve "bizarre"que les palets "reprennent leur position initiale en même temps alors qu'au départ [place la vidéo au début] quand l'observateur se positionne à l'horizontale, ben, on a l'impression que, euh, les deux palets, euh, / n'ont pas la même vitesse "et conclut que "selon la position de l'observateur, et ben, euh, / on n'aura pas la même vision, des choses". $\mathrm{AB}$, quant à elle, observe l'écran en pause (vidéo 2) : le palet rouge apparaît devant le palet jaune et commente: "Mais en fait au tout début on voit directement un décalage. Dès le début, en fait je vous disais, j'ai l'impression qu'ils partent pas en même temps". Elle relance la vidéo pour observer s'il y a décalage dès le début et poursuit : "Mais en fait si // Ah ben c'est justement c'est le fait qu'on reçoit les rayons émis par le palet jaune, après. Et donc du coup on a l'impression qu'il est en retard".

Le plan travelling provoque souvent le même jeu de relance : cette fois, l'attention est retenue par les scénaristes de la vidéo (c'est-à-dire les auteurs de cette étude) mais de la même manière, les causes sont recherchées dans l'histoire passée des objets et des observateurs de la scène, histoire dont les étudiants ne conservent pas de souvenir précis dans la mesure où ils ne savent pas a priori ce qui doit retenir leur attention. À ce sujet, KdF a indiqué la difficulté qu'il avait à se souvenir du sens du mouvement des palets lorsqu'il effectue une pause lors d'un travelling, ce que JT a également éprouvé.

Les moments choisis pour les mises en pause correspondent en général aux moments où les phénomènes (décalage des collisions avec la table de billard, étirement et inclinaison des palets) sont les plus accentués, par exemple, lorsque l'observateur est situé à l'une des extrémités de la table (vidéo 2 - décalage des collisions), ou lorsque le phénomène disparaît, par exemple lorsque les palets démarrent et se croisent au même instant au milieu de la table (vidéo 2 et vidéo 3) alors qu'ils arrivent de manière décalée au bord de la table, ou encore, lorsque le point de vue anéantit les déformations (par exemple, lorsque les palets sont vus du dessus dans la vidéo 5).

\section{Discussion et conclusion}

Notre exploration avait pour objet de déterminer les éléments conceptuels accessibles par le biais des six scènes relativistes présentées sous forme de vidéos 2D. Plus particulièrement, nous avons cherché à savoir d'une part si les angles de vues et les déplacements des observateurs étaient adaptés à l'identification d'effets à expliquer, et 
d'autre part, si les effets visibles permettaient la mobilisation d'éléments conceptuels relevant de la physique de la lumière et de la cinématique relativiste.

Une façon de déterminer si les vidéos remplissent le rôle que nous leur avons assigné, consiste à vérifier si le phénomène visé a été identifié dans la vidéo dédiée. C'est bien le cas pour les deux premières vidéos ( 2 et 3 ), par contre pour les vidéos 4 et 5 les résultats sont plus contrastés puisque cinq étudiants seulement ont décrit les déformations dans la vidéo 4 et sept étudiants ont identifié l'inclinaison des palets avant la vidéo 5 . Pour certains, revoir un phénomène dans la vidéo suivante leur permet d'en prendre conscience. De plus les descriptions fournies manquent de précision. Nous pouvons invoquer plusieurs raisons : les vidéos 4 et 5 comportent des travellings pour lesquels certains étudiants ont quelques difficultés à repérer la position de l'observateur ou le sens du mouvement; les déformations et inclinaisons des palets sont visibles dans les vidéos précédentes et ont pu être repérées avant. En revanche, les manipulations permises par la simulation semblent contribuer à l'amorce d'explications engageant un ou plusieurs éléments conceptuels pertinents : l'observation d'une image figée à un instant où l'effet mis en scène est accentué (mise en pause) conduit à remonter l'histoire ayant conduit à cet effet (relance).

Notre deuxième interrogation concerne les éléments conceptuels accessibles par le biais des vidéos. De façon globale, quel que soit le phénomène identifié dans les vidéos 2 à $5, R G$ et SP n'ont pas réussi à formuler d'explication, ce qu'on peut relier à leur façon insuffisante d'exprimer la vision d'un objet. SL et PEMS en ont donné une seule, les autres entre trois et quatre. Environ la moitié des étudiants interrogés a utilisé les éléments conceptuels attendus à bon escient pour les vidéos 2 à 5 . La qualité (au sens explicitation et articulation de tous les paramètres pertinents) des explications est satisfaisante pour le phénomène décalage, et diminue pour les autres, vitesse, déformation et inclinaison. En effet bien que ces explications comportent des éléments conceptuels communs, elles incluent plus de grandeurs variables que l'explication du décalage.

L'explication du décalage des collisions met en jeu un raisonnement incluant la vitesse de propagation de la lumière qui modifie la date à laquelle on perçoit ces collisions. II n'y a qu'une grandeur variable à prendre en compte pour chaque palet, la distance entre le point d'impact et l'observateur qui permet de déduire la durée de propagation de l'information lumineuse dans chaque cas.

L'explication des vitesses différentes inclut quatre paramètres à articuler, la distance parcourue par le palet et la durée mise pour la parcourir, la distance parcourue par la lumière et la durée de propagation de l'information lumineuse. Moins d'étudiants parviennent à exprimer clairement leur pensée. Les raisonnements impliquant plusieurs grandeurs variant en même temps sont plus ardus à mener à leur terme (Viennot, 1992).

L'explication des déformations et de l'inclinaison nécessite de considérer les palets comme un ensemble de points n'étant pas perçus à la même date par l'observateur. À nouveau plusieurs variables interviennent, distance d'un point à l'observateur, 
vitesse du point par rapport à la table, durée de propagation de la lumière d'un point à l'observateur et ce pour chacun des deux points (ou partie) extrêmes du palet. À la difficulté de raisonner avec plusieurs grandeurs covariantes s'ajoute celle de le faire pour deux points d'un même palet. Les étudiants ont beau dire que la raison est la même, "une histoire de différences de distances", ils peinent à dire pourquoi cette même raison dans un cas se traduit par un décalage temporel et dans un autre cas par un décalage de forme. La nécessité de discrétiser l'objet " palet " en considérant à un instant donné chacun des points du palet comme un événement semble une difficulté réelle. C'est une difficulté semblable qu'avait identifiée Buty (2000) dans une étude visant à évaluer l'impact d'une modélisation informatique sur l'apprentissage de concepts de l'optique géométrique. Les manipulations et les productions des élèves utilisateurs de l'environnement informatique révélaient une prégnance forte d'une vision holistique des objets lumineux à la source des raisonnements de type "image voyageuse" (Viennot, 2002).

La passation de la vidéo 6 n'a pas du tout produit l'explication attendue. Aucun étudiant n'imagine qu'on ait pu enlever la propagation de la lumière de la simulation pour obtenir les palets floutés. Cela peut s'expliquer de plusieurs façons. Dans la mesure où les deux questions préalables orientent toutes les explications précédentes (pour ceux qui ont compris ce qui était en jeu) vers la prise en compte de la propagation de la lumière, ne plus devoir le faire relève quasiment d'une rupture de contrat didactique. En effet la consigne était vague à dessein et ne laissait pas supposer que les concepteurs de la simulation aient pu aller jusqu'à cette extrémité. Par ailleurs les connaissances de relativité restreinte des étudiants sont absentes ou quasi oubliées, ce qui ne favorise pas la prise de conscience que la règle qui prévalait dans les vidéos précédentes ait pu être complètement modifiée. Le potentiel de cette vidéo est cependant réel dans la mesure où KdF qui n'a pas étudié la relativité restreinte revient aussitôt sur l'interprétation erronée des palets floutés qu'il a donnée. Il est toutefois probable que le parti-pris d'un guidage faible ne soit pas du tout adapté pour l'exploitation de cette vidéo-là dans la mesure où l'explication n'est pas une simple mobilisation de connaissances déjà présentes convenablement articulées (ce qui semblait convenir à un guidage faible).

Il convient donc de revoir le protocole et dire à quoi correspondent les palets floutés (pas de propagation de lumière jusqu'à l'observateur) et demander de décrire et d'interpréter les différences entre les palets floutés et les autres. Cela permettrait à ceux qui n'ont pas perçu le rôle de la propagation de la lumière de revenir dessus et de reprendre un cycle d'explication. Cependant il parait difficile de faire découvrir la contraction des longueurs à quelqu'un qui n'a jamais étudié la relativité restreinte. Et ceci est d'autant plus difficile que, vidéo 6 mise à part, les effets minkovskiens ne sont pas directement "visualisables" dans la mesure où ils s'associent aux effets liés à la prise en compte du délai de propagation de la lumière entre l'objet et l'œil de l'observateur. L'objectif de notre simulation est de rendre manifestes les effets relativistes mais ceux-ci conjuguent deux effets qui méritent d'être dissociés visuellement 
mais dont l'appropriation ne peut pas être modalisée de la même manière. Les étudiants ont vu leur attention portée vers les effets non minkovkiens pendant la quasi-totalité de la simulation sans que soient mentionnés les effets minkovskiens (ou à mauvais escient). La visualisation soudaine des palets contractés (c'est-à-dire fantômes) nécessite un changement radical de registre explicatif, qui engage un savoir non connu des étudiant et non accessible par la simple visualisation. L'une des conséquences majeures de notre étude est de revoir, d'une part, l'ordre de passation des vidéos et, d'autre part, de revoir l'accompagnement qui soutient la visionnage des vidéos en fonction des effets manifestes, de manière à aider les étudiants à discriminer les effets minkovskiens des effets liés à la propagation de la lumière de l'objet à l'œil de l'observateur.

La vidéo 6 pourrait venir juste après la vidéo 1 afin de mettre d'emblée l'accent sur les déformations intrinsèques aux effets relativistes d'une part et sur le rôle joué par la propagation de la lumière d'autre part, et il faudrait revoir le rôle du chercheur-intervieweur pour qu'il introduise des éléments de guidage plus fort selon une modalité qui reste à préciser en s'appuyant sur les résultats de cette étude exploratoire. Cette nouvelle étude pourrait être effectuée de façon pilote auprès d'étudiants volontaires de master MEEF à nouveau. Ensuite, selon les résultats obtenus, il pourrait être possible de proposer une formation intégrée dans le cursus du master, avec un double objectif, compléter (ou apporter) une formation disciplinaire spécifique sur la relativité restreinte et faire prendre conscience de la difficulté à distinguer les effets dus à la propagation de la lumière des effets minkovskiens.

\author{
Clément Maisch \\ clement.maisch@upmf-grenoble.fr \\ Isabelle Kermen \\ isabelle.kermen@univ-artois.fr \\ Cécile de Hosson \\ cecile.dehosson@univ-paris-diderot.fr \\ Étienne Parizot \\ parizot@apc.univ-paris-diderot.fr
}

\title{
Bibliographie
}

ADAMS W.K., PERKINS K.K., PODOLEFSKY N.S., DUBSON M., FINKELSTEIN N.D. $\&$ WIEMAN C.E. (2006) New instrument for measuring student beliefs about physics and learning physics: The Colorado Learning Attitudes about Science Survey. Physical Review Special Topics-Physics Education Research, $\mathrm{n}^{\circ} 2$ (010101), p. 1-14

BEAUFILS D. \& RICHOUX B. (2003). Un schéma théorique pour situer les activités avec des logiciels de simulation dans l'enseignement de la physique. Didaskalia, $\mathrm{n}^{0} 23$, p. 9-38. 
BUTY C. (2000). Étude d'un apprentissage dans une séquence d'enseignement en optique géométrique à l'aide d'une modélisation informatique. Thèse de doctorat. Lyon : université de Lyon 1.

BUTY C. (2002). Modelling in Geometrical Optics Using a Microcomputer. In D. Psillos $\& \mathrm{H}$. Niedderer (éd.), Teaching and Learning in the Science Laboratory, Berlin : Springer, p. 231-242.

CHAUVET F. (1996) Teaching Colour: Designing and evaluation of a sequence, European Journal of Teacher Education, vol. 19, n², p. 119-134.

CHAUVET F. \& DUPREZ C. (2003). Environnement d'apprentissage fondé sur une simulation: appropriation par les enseignants stagiaires d'IUFM. $3^{e}$ rencontres scientifiques de l'ARDIST, Toulouse. p. 13-20.

DOAT T., PARIZOT E. \& VEZIEN J. M. (2011) A carom billiard to understand special relativity. In M. Hirose, B. Lok, A. Majumder, D. Schmalstieg (éd.), IEEE Virtual Reality Conference, VR 2011, p. 201-202.

FINKELSTEIN N. D., ADAMS W. K., KELLER C. J., KOHL P. B., PERKINS K. K., PODOLEFSKY N. S., REID S. \& LEMASTER R. (2005). When learning about the real world is better done virtually: A study of substituting computer simulations for laboratory equipment. Physical Review Special Topics-Physics Education Research, vol. 1, $\mathrm{n}^{0} 1$ (010103).

GUESNE E. (1984). Children's idea about light / les conceptions des enfants sur la lumière. New Trends in Physics Teaching, vol. 4, Paris, UNESCO, p. 179-192.

HOSSON C. de, KERMEN I. \& PARIZOT E. (2010). Exploring students' understanding of reference frames and time in Galilean and special relativity. European Journal of Physics, $n^{0} 31$, p. 1527-1538.

HOSSON C. de, DOAT T., KERMEN I. \& VÉZIEN J. M. (2012). Designing learning scenarios for a 3D virtual environment: The case of special relativity. Latin-American Journal of Physics Education, vol. 1, n²7, p. 6.

MAISCH C., DE HOSSON C. \& KERMEN I. (2012). Étude exploratoire d'un scénario d'utilisation pour un environnement virtuel en 3D dans le cas de la relativité

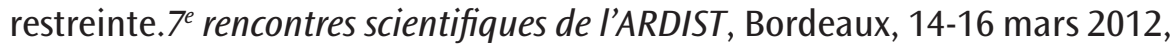
p. 261-269.

MELLAR H. \& BLISS J. (1994). Introduction: Modelling and Education. In H. Mellar, J. Bliss, R. Boohan, J. Ogborn \& C. Tompsett (éd.), Learning with artificial worlds: computerbased modelling in the curriculum, Londres: The Falmer Press, p. 1-7.

MEN (ministère de l'Éducation nationale) (2011). Bulletin officiel de l'Éducation nationale, spécial $n^{\circ} 8$ du 13 octobre 2011. 
RICHOUX C., SALVETAT C. \& BEAUFILS D. (2002). Simulation numérique dans l'enseignement de la physique : enjeux, conditions. Bulletin de l'Union des physiciens, vol. $96, \mathrm{n}^{\circ} 842$, p. 497-521.

RUDER H., WEISKOPF D., NOLLERT H.S. \& MÜLLER T. (2008). How computers can help us in creating an intuitive access to relativity. New Journal of Physics, $\mathrm{n}^{0} 10$.

SALTIEL E. \& MALGRANGE J.L. (1980). Spontaneous ways of reasoning in elementary kinematics. European Journal of Physics, n ${ }^{0} 1$, p. 73-80.

SAVAGE C. M., SEARLE A. \& MCCALMAN L. (2007). Real Time Relativity: exploration learning of special relativity. American Journal of Physics, nº75, p. 791-798.

SCHERR R., SCHAFFER P. \& VOKOS S. (2001). Student understanding of time in special relativity: simultaneity and references frames. American Journal of Physics, $\mathrm{n}^{\circ} 69$, p. 24-35.

VAN SOMEREN M. W., BARNARD Y. F., \& SANDBERG J. A. (1994). The think aloud method: A practical guide to modelling cognitive processes, vol. 2. Londres : Academic Press.

VIENNOT L. (1992). Raisonnement à plusieurs variables: tendances de la pensée commune. Aster, nº14, p. 127-140.

VIENNOT L. (2002). Enseigner la physique. Paris/Bruxelles : De Boeck.

VILLANI A. \& PACCA J.L.A. (1987). Students' spontaneous ideas about the speed of light. International Journal of Science Education, vol. 9, nº 1, p. 55-66.

VINCE J. \& TIBERGHIEN A. (2000). Simuler pour modéliser: Le cas du son. Sciences et techniques éducatives, vol. 7, $\mathrm{n}^{\circ} 2$, p. 333-366.

WEISKOPF D., BORCHERS M., ERTL T., FALK M., FECHTIG 0., FRANK R., GRAVE F., KING A., KRAUS U., MÜLLER T., NOLLERT H.-P, RICA MENDEZ I., RUDER H., SCHAFHITZEL T., SCHÄ S., ZAHN C. \& ZATLOUKAL M. (2006). Explanatory and Illustrative Visualization of Special and General Relativity. IEEE Transactions on Visualization and Computer Graphics, vol. 12, nº 4, p. 522-534. 


\section{Annexes}

\section{Annexe 1 : présentation des six vidéos de l'expérimentation, positions de la caméra et descriptions des effets}

\section{Vidéo 1}

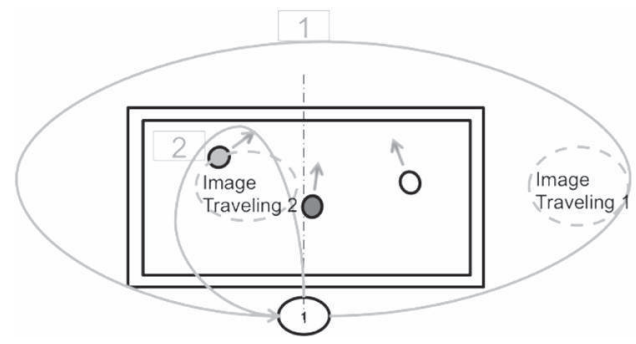

Fig. 3 : mouvements et positions de caméra de la vidéo 1
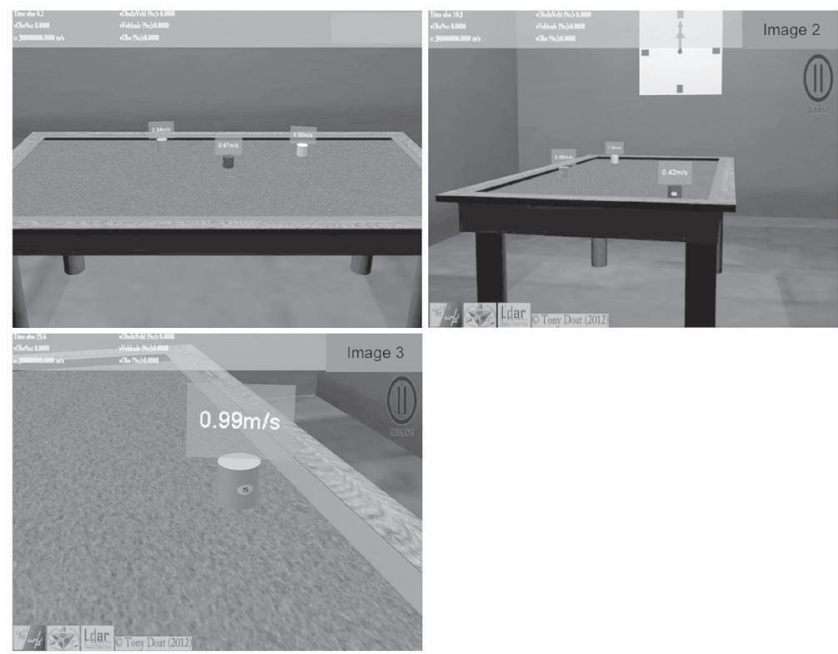

Fig. 4 : captures d'écran de la vidéo 1 pour les positions d'observation

Trois palets se déplacent sans frottement sur la table de billard ; la vitesse de la lumière est fixée à $300000 \mathrm{~km} / \mathrm{s}$, celle des palets est de l'ordre de $1 \mathrm{~m} / \mathrm{s}$. L'observateur observe la scène avec un petit recul par rapport à la table (pendant $10 \mathrm{~s}$, image 1), puis la scène est " gelée ", dans le sens où tout s'arrête sur la table du billard, et l'observateur effectue le tour de la table de billard pour observer la scène sous tous les angles (image 2, pendant 12 s). Ensuite la scène est relancée et au bout de $24 \mathrm{~s}$ un nouveau plan travelling est effectué permettant de tourner autour d'un seul palet pour observer sa forme (pendant $10 \mathrm{~s}$, image 3). Finalement la scène est relancée jusqu'à la fin de la vidéo (pendant 14 s). 


\section{Vidéo 2}

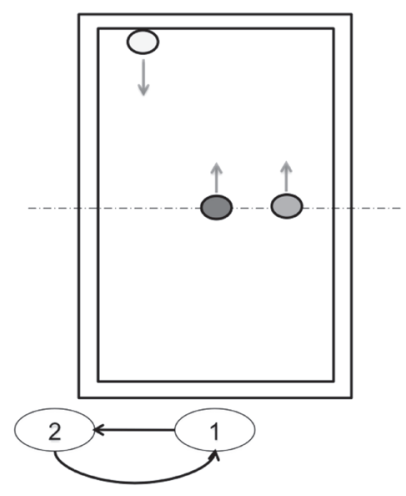

Fig. 5 : mouvements et positions de caméra de la vidéo 2
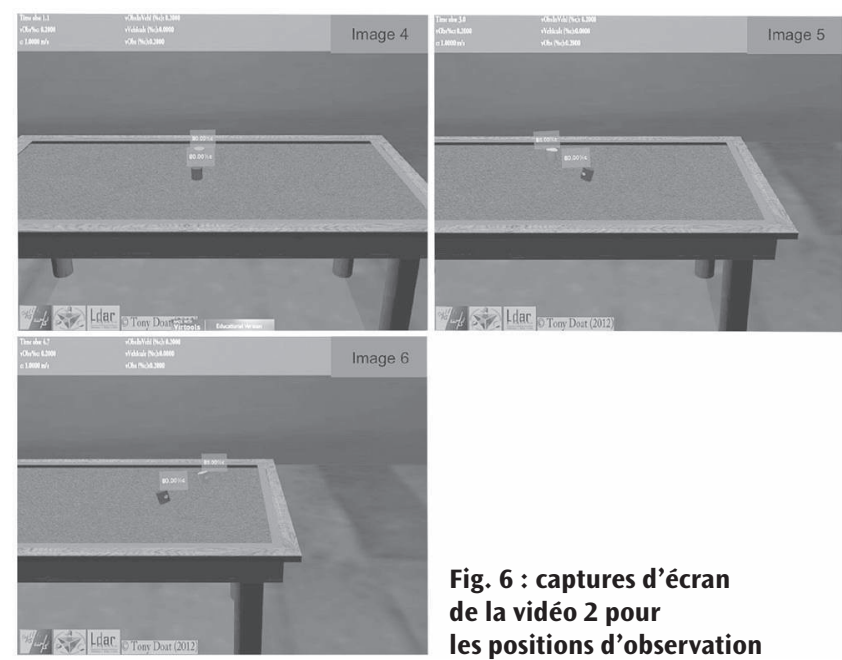

Fig. 6 : captures d'écran de la vidéo 2 pour les positions d'observation

Cette vidéo montre le déplacement de deux palets partant au même instant du centre du billard. L'observateur après avoir vu le départ des palets de la vidéo (position 1, image 4) se déplace le long de la table (position 2, $6 \mathrm{~s}$, image 5) jusqu'au coin droit (position 3). Là, il s'arrête et regarde la situation pendant $10 \mathrm{~s}$ puis revient à sa position initiale (en $5 \mathrm{~s}$ ). Pour finir il regarde encore $10 \mathrm{~s}$ la situation dans la position 1 (image 6).

\section{Vidéo 3}

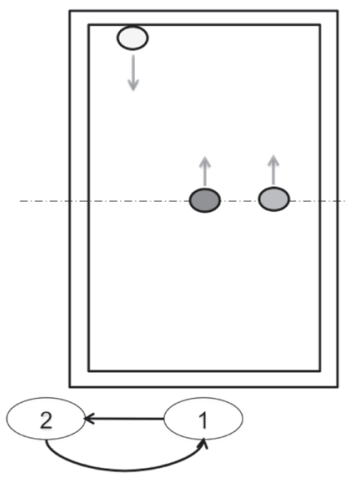

Fig. 7 : mouvements et positions de caméra de la vidéo 3
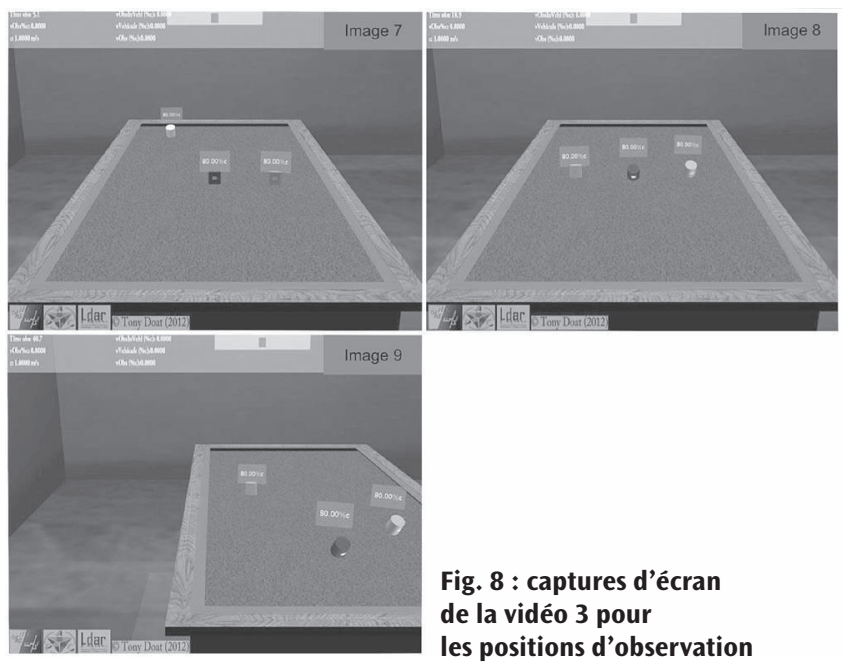

Fig. 8 : captures d'écran

de la vidéo 3 pour les positions d'observation 
Cette vidéo montre le déplacement de trois palets sur la table de billard. Deux palets (rouge et orange) partent au même instant du centre du billard en s'éloignant de la caméra. Le troisième palet (palet jaune) part du bord opposé à celui où se trouve la caméra et avance vers elle. La caméra est placée sur le bord court du billard, c'est-à-dire le bord où les palets rebondissent. Dans sa première position (position 1, image 7), elle se situe au centre du bord du billard pendant $20 \mathrm{~s}$, puis elle se déplace vers le coin gauche (position 2, image 9). Au bout de 20 s dans cette position elle revient à sa position initiale (position 1) jusqu'à la fin de la vidéo (pendant 20 s). Chacun des déplacements prend environ 3 secondes. Dans la position 1 , l'observateur peut voir les palets se déplacer en sens contraire et prendre des formes et des vitesses différentes (image 8).

\section{Vidéo 4}

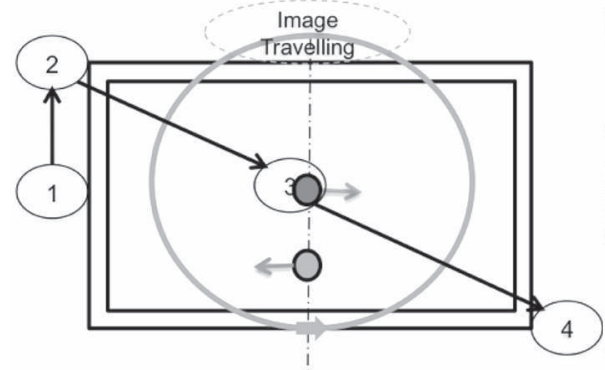

Fig. 9 : mouvements et positions de caméra de la vidéo 4

Fig. 10 : captures d'écran de la vidéo 4 pour les positions d'observation

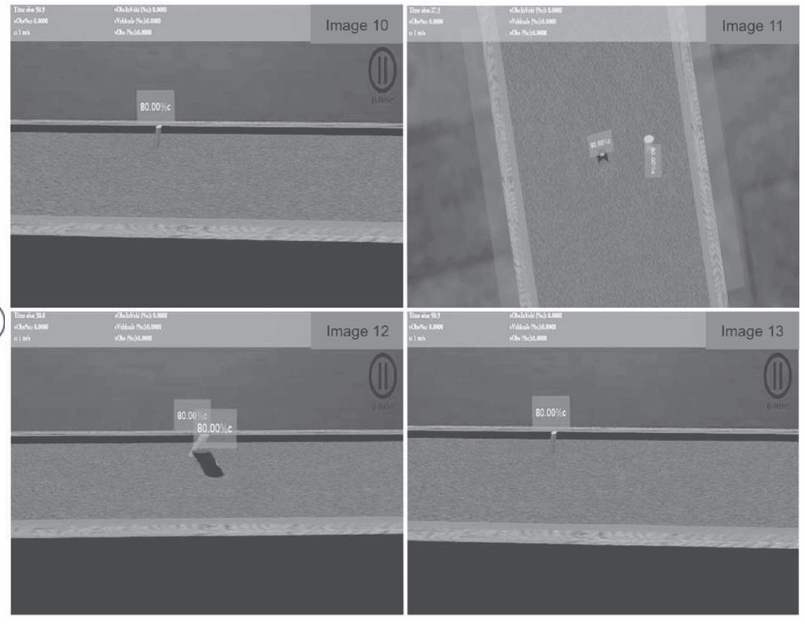

La caméra fait le tour des palets avant leur mise en mouvement à l'aide d'un plan travelling. Les palets sont ensuite mis en mouvement au même instant, dans des sens opposés : la caméra est placée en position 1. Après $20 \mathrm{~s}$, elle se déplace sur le côté gauche (position 2). À $27 \mathrm{~s}$, la situation est mise en pause et un plan travelling est effectué autour des palets (image 10) puis le mouvement des palets est relancé. La caméra se positionne ensuite au-dessus du billard puis s'arrête (position 3, image 11) et un nouveau plan travelling est effectué (image 12). La situation est relancée et l'observateur atteint le coin opposé (position 4). Une nouvelle pause est effectuée avec un plan travelling autour du palet jaune (image 13). 


\section{Vidéo 5}

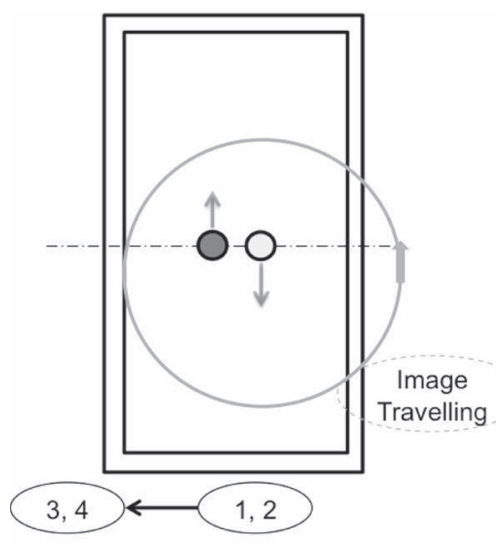

Fig. 11 : mouvements et positions de caméra de la vidéo 5

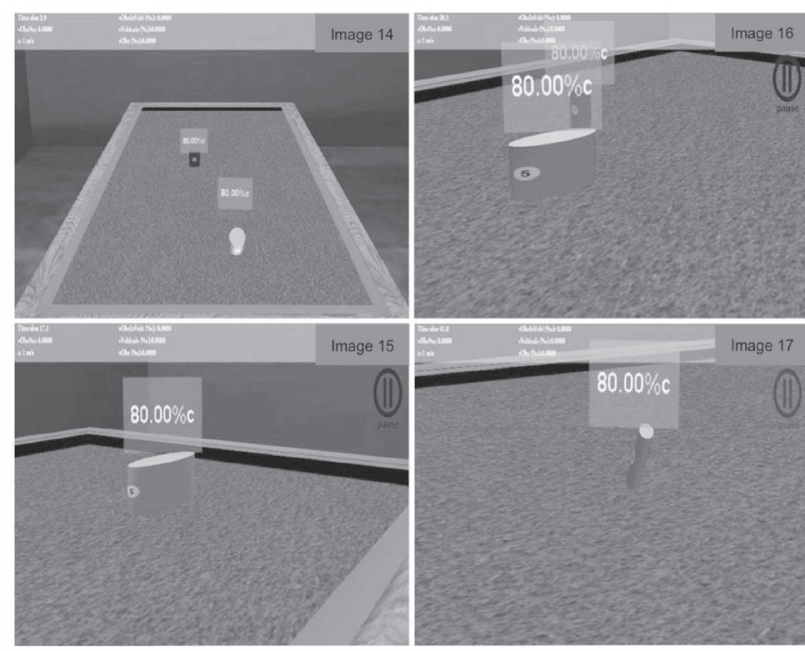

Fig. 12 : captures d'écran de la vidéo 5 pour les positions d'observation

Cette vidéo présente le déplacement de deux palets partant aux mêmes instants dans des directions opposés, à la même vitesse (par rapport à la table). À l'instant où les palets se mettent en mouvement, la caméra se situe au milieu d'une des petites bandes, au-dessus de la table (position 1, image 14). Après le premier rebond de chaque palet avec chaque bord de la table, la caméra se place au niveau du billard (position 2) : les palets rebondissent une fois et un plan travelling est effectué autour du palet orange qui vient vers l'observateur (image 15, travelling 1). La situation est relancée et après deux rebonds des palets, la caméra se place au coin droit de la bande toujours au niveau de billard (position 3). Après deux rebonds de chacun des palets, un second plan travelling est effectué (image 16, travelling 2). Les palets sont relancés (56 s) et rebondissent encore trois fois sur la bande avant que la caméra ne se repositionne au-dessus de la table (à hauteur d'homme, position 4). Après deux rebonds des palets, un nouveau plan travelling est effectué sur le palet jaune qui cette fois-ci s'éloigne de l'observateur (image 17, travelling 3). 
Vidéo 6
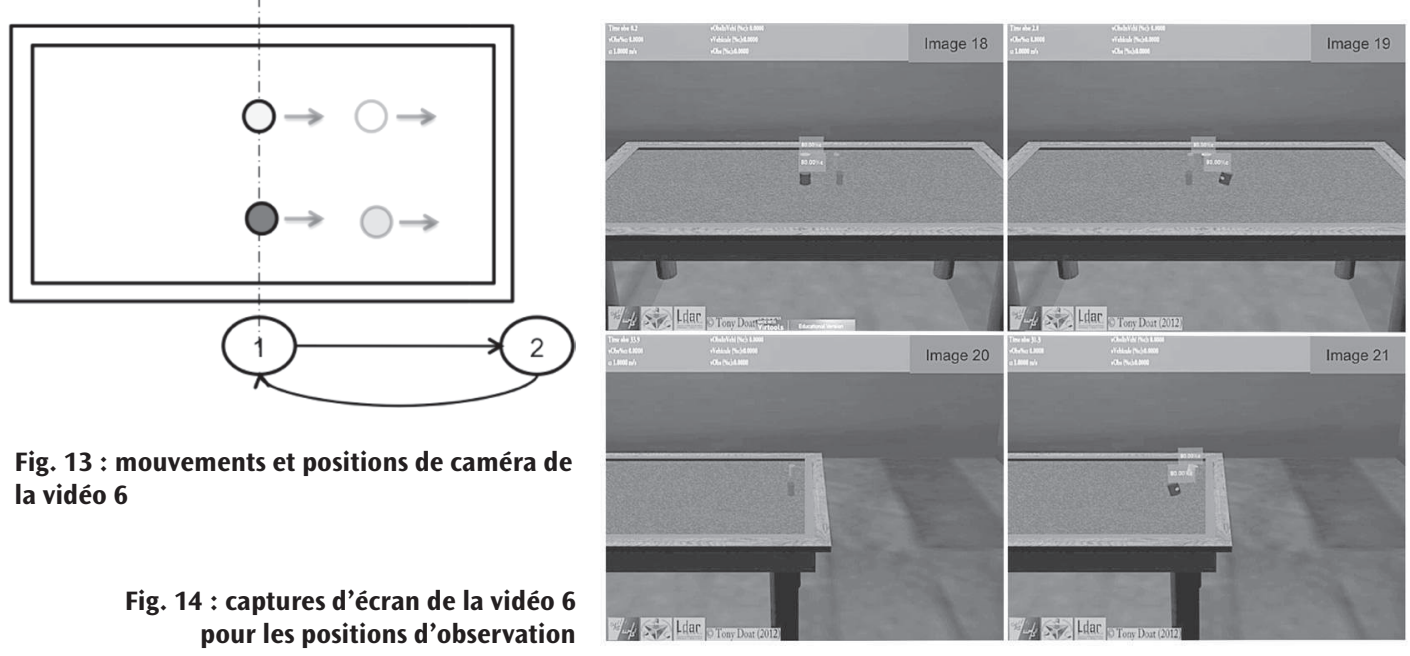

Fig. 13 : mouvements et positions de caméra de la vidéo 6

Fig. 14 : captures d'écran de la vidéo 6 pour les positions d'observation

Cette vidéo est similaire à la vidéo 2 mais deux palets " fantômes " ont été ajoutés et sont visibles en transparence.

L'observateur dans la position 1, au centre la bande, voit donc les palets " fantômes " partir dès le lancement de la vidéo (image 18). Après un instant ( $3 \mathrm{~s}$ ), les palets " réels " se mettent en mouvement. Alors que les palets " fantômes " ne montrent aucune déformation, les palets " réels "sont inclinés dans le sens de leur déplacement (image 19). La caméra enregistre le mouvement et la forme des palets "réels " et " fantômes " sur quelques rebonds sur la bande. Elle se déplace ensuite sur le côté droit (position 2). On constate alors que les palets " fantômes " rebondissent sur la bande au même instant (image 20) alors que les palets "réels " touchent la table à deux instants différents (image 21), exactement comme dans la vidéo 2. La caméra revient en position 1 et l'on observe le déplacement des palets encore quelques secondes avant la fin de la vidéo. 


\section{Annere 2}

\begin{tabular}{|c|c|c|c|c|c|c|c|c|c|c|}
\hline & & \multicolumn{9}{|c|}{ Face aux vidéos 2 à 5} \\
\hline & Phénomène décrit/étudiant & $\mathrm{AB}$ & YS & RG & JT & $\mathrm{KdF}$ & JAD & SP & PEMS & SL \\
\hline \multirow{6}{*}{ 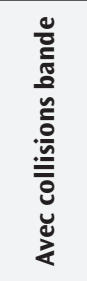 } & collisions avec bande non simultanées & 1 & 1 & 2 & 1 & 0 & 1 & 0 & 1 & 0 \\
\hline & décalage collisions dépend position observateur & 1 & 0 & 1 & 1 & 0 & 0 & 0 & 0 & 0 \\
\hline & collisions simultanées si observateur en face & 0 & 0 & 0 & 1 & 0 & 0 & 0 & 0 & 0 \\
\hline & palet éloigné en retard sur palet proche & 1 & 1 & 0 & 0 & 2 & 1 & 0 & 1 & 0 \\
\hline & $\begin{array}{l}\text { palet proche semble aller plus vite que palet } \\
\text { éloigné }\end{array}$ & 0 & 0 & 0 & 0 & 0 & 0 & 0 & 0 & 1 \\
\hline & palet rouge part d'abord & 1 & 0 & 0 & 0 & 1 & 0 & 0 & 1 & 0 \\
\hline \multirow{2}{*}{ Vitesse } & palet rapide en venant vers observateur & 2 & 1 & 0 & 1 & 1 & 1 & 2 & 1 & 1 \\
\hline & palet lent en s'écartant de l'observateur & 2 & 1 & 0 & 1 & 1 & 1 & 2 & 1 & 1 \\
\hline \multirow{4}{*}{ 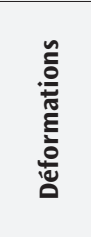 } & $\begin{array}{l}\text { palet allongé en s'approchant de } \\
\text { l'observateur }\end{array}$ & 1 & 1 & 0 & 1 & 1 & 1 & 0 & 0 & 0 \\
\hline & palet contracté en s'éloignant de l'observateur & 1 & 1 & 0 & 1 & 1 & 1 & 0 & 0 & 0 \\
\hline & forme des palets différente & 0 & 0 & 1 & 0 & 0 & 0 & 1 & 0 & 0 \\
\hline & $\begin{array}{l}\text { déformation palet change selon position } \\
\text { observateur }\end{array}$ & 0 & 0 & 0 & 2 & 1 & 0 & 0 & 1 & 0 \\
\hline \multirow{6}{*}{ 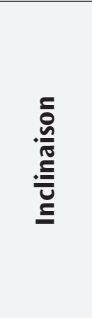 } & palet incliné par rapport verticale & 0 & 1 & 0 & 0 & 1 & 0 & 1 & 1 & 0 \\
\hline & sens inclinaison change après collision bande & 0 & 1 & 0 & 0 & 0 & 0 & 0 & 0 & 0 \\
\hline & $\begin{array}{l}\text { inclinaison du palet dans le sens du } \\
\text { mouvement }\end{array}$ & 0 & 1 & 0 & 1 & 0 & 1 & 0 & 0 & 0 \\
\hline & palet pas penché si observateur baissé & 0 & 1 & 0 & 0 & 0 & 0 & 0 & 0 & 0 \\
\hline & allongé selon axe observation & 0 & 0 & 0 & 0 & 1 & 1 & 0 & 0 & 0 \\
\hline & $\begin{array}{l}\text { face supérieure du palet décalée par rapport } \\
\text { à face inférieure }\end{array}$ & 1 & 0 & 0 & 1 & 0 & 1 & 0 & 0 & 1 \\
\hline
\end{tabular}

Tableau 9 : éléments descriptifs des phénomènes

Le chiffre figurant dans chaque case correspond au nombre de fois où l'étudiant a fait la description.

\section{Annere 3}

\begin{tabular}{|l|c|c|c|c|c|c|c|c|c|}
\hline & $\mathrm{AB}$ & $\mathrm{YS}$ & $\mathrm{RG}$ & $\mathrm{JT}$ & $\mathrm{KdF}$ & $\mathrm{JAD}$ & $\mathrm{SP}$ & $\mathrm{PEMS}$ & $\mathrm{SL}$ \\
\hline Vidéo 2 & 6 & 1 & 2 & 1 & 2 & 2 & 6 & 8 & 3 \\
\hline Vidéo 3 & 3 & 2 & 7 & 2 & 3 & 1 & 2 & 7 & 2 \\
\hline Vidéo 4 & 4 & 2 & 7 & 3 & 4 & 3 & 3 & 4 & 4 \\
\hline Vidéo 5 & 2 & 2 & 2 & 2 & 2 & 2 & 1 & 3 & 2 \\
\hline Vidéo 6 & & & & 6 & 4 & 5 & 4 & 9 & 4 \\
\hline
\end{tabular}

Tableau 10 : total des visualisations de chaque vidéo par étudiant

\begin{tabular}{|l|c|c|c|c|c|c|c|c|c|}
\hline & $\mathrm{AB}$ & $\mathrm{YS}$ & $\mathrm{RG}$ & $\mathrm{JT}$ & $\mathrm{KdF}$ & $\mathrm{JAD}$ & $\mathrm{SP}$ & $\mathrm{PEMS}$ & $\mathrm{SL}$ \\
\hline Vidéo 2 & 2 & 0 & 3 & 0 & 2 & 1 & 0 & 0 & 0 \\
\hline Vidéo 3 & 5 & 1 & 3 & 0 & 3 & 3 & 0 & 0 & 0 \\
\hline Vidéo 4 & 1 & 9 & 7 & 9 & 11 & 4 & 6 & 1 & 2 \\
\hline Vidéo 5 & 1 & 1 & 2 & 5 & 7 & 5 & 1 & 0 & 0 \\
\hline Vidéo 6 & & & & 9 & 5 & 4 & 0 & 2 & 0 \\
\hline
\end{tabular}

Tableau 11 : nombre de pauses faites par vidéo par étudiant 